\title{
Leveraging CD39 To Identify Tumor-Reactive CD8 T cells In Human Lung Cancer
}

Andrew Chow ${ }^{1,2,3}$, Fathema Z. Uddin ${ }^{1}$, Levi Mangarin², Hira Rizvi ${ }^{4}$, Anton Dobrin ${ }^{5,6}$, Sam Tischfield ${ }^{7}$, Alvaro Quintanal-Villalonga1 ${ }^{1}$, Joseph M. Chan ${ }^{1,3}$, Nisargbhai Shah ${ }^{1}$, Viola Allaj ${ }^{1}$, 5 Parvathy Manoj ${ }^{1}$, Marissa Mattar ${ }^{8}$, Maximiliano Meneses ${ }^{8}$, Rebecca Landau ${ }^{8}$, Mariana Ward ${ }^{8}$, Amanda Kulick ${ }^{8}$, Charlene Kwong ${ }^{8}$, Matthew Wierzbicki ${ }^{8}$, Jessica Yavner ${ }^{8}$, Shweta S. Chavan ${ }^{7}$, Abigail Farillas ${ }^{1}$, Michael Liu ${ }^{1}$, Aliya Holland ${ }^{2}$, Metamia Ciampricotti ${ }^{1}$,

${ }^{1}$ Thoracic Oncology Service, Department of Medicine, Memorial Sloan Kettering Cancer Center, 13 USA

$14{ }^{2}$ Ludwig Collaborative and Swim Across America Laboratory, Memorial Sloan Kettering Cancer 15 Center, New York, NY, USA

$16{ }^{3}$ Department of Medicine, Weill Cornell Medical College, USA

$17{ }^{4}$ Druckenmiler Center for Lung Cancer Research, Memorial Sloan Kettering Cancer Center, USA

$18{ }^{5}$ Center for Cell Engineering, Memorial Sloan Kettering Cancer Center, USA

19 'Louis V. Gerstner, Jr. Graduate School of Biomedical Sciences, Memorial Sloan Kettering

20 Cancer Center, USA

$21{ }^{7}$ Marie-Josée and Henry R. Kravis Center for Molecular Oncology, Memorial Sloan Kettering 22 Cancer Center, USA

$23{ }^{8}$ Antitumor Assessment Core, Memorial Sloan Kettering Cancer Center, USA

249 Department of Epidemiology \& Biostatistics, Memorial Sloan Kettering Cancer Center, USA

$25{ }^{10}$ Breast Medicine Service, Department of Medicine, Memorial Sloan Kettering Cancer Center, 26 USA

$27{ }^{11}$ Human Oncology and Pathogenesis Program, Memorial Sloan Kettering Cancer Center, USA

$28{ }^{12}$ Parker Institute for Cancer Immunotherapy, Memorial Sloan Kettering Cancer Center, USA

$29{ }^{13}$ Present Address: Oncology R\&D, Astra-Zeneca, USA

$30{ }^{14}$ Molecular Pharmacology Program, Memorial Sloan Kettering Cancer Center, USA

$31{ }^{15}$ These senior authors contributed equally to this work.

32 *Lead Contact

33 Correspondence: merghout@mskcc.org (TM) and rudinc@mskcc.org (CMR) 


\section{SUMMARY}

35 CD39 has emerged as a marker of tumor-reactive CD8 T cells. Using single-cell

36 CITE/RNA/TCRseq, we show that CD39+ CD8 T cells in human lung cancers demonstrate

37 transcriptional and proteomic features of exhaustion, tumor reactivity, and clonal expansion. Flow

38 cytometry of 440 lung cancer specimens revealed that CD39 level on CD8 T cells is only weakly

39 correlated with tumoral features, such as histology, driver mutation, PD-L1 and tumor mutation

40 burden. CD8 T cells reactive against EGFR driver mutations preferentially expressed CD39,

41 suggesting that CD39 can be leveraged to identify neoantigen-reactive CD8 T cells. PD-1 axis

42 blockade, but not cytotoxic chemotherapy, increased intratumoral CD39+ CD8 T cells. CD39

43 correlated with PD-1 expression on CD8 T cells and high pre-treatment/early-on-treatment levels

44 were associated with improved clinical outcomes from immune checkpoint blockade. This

45 extensive and comprehensive profiling of the clinical, pathological and molecular features

46 highlights the utility of CD39 as a proxy for tumor-reactive CD8 T cells in human lung cancer.

\section{KEYWORDS}

49 Immune-checkpoint blockade, non-small cell lung cancer, T cell receptors, tumor mutation 50 burden, CD8 T cells 


\section{INTRODUCTION}

53 Lung cancer is the leading cause of cancer death in the world. Immune checkpoint blockade (ICB)

54 utilizing antibodies that block programmed cell death protein 1 (PD-1), programmed death-ligand

551 (PD-L1), and cytotoxic T-lymphocyte-associated protein 4 (CTLA-4) has ushered in a new era

56 of hope, with long-term durable disease control in a subset of patients with lung cancer $(1,2)$.

57 However, most patients do not respond to ICB therapy and many of those who initially respond

58 eventually develop recurrent and progressive disease. While PD-L1 on tumor cells and tumor

59 mutation burden (TMB) have been validated as predictors of benefit from ICB in lung cancer (3,

60 4), there is an opportunity to further refine these biomarkers by incorporating features of CD8 T

61 cells, which are critical mediators of ICB efficacy.

63 Despite early suggestions that measurement of bulk CD8 tumor infiltrating lymphocytes (TILs)

64 would predict benefit from ICB (5), multiple recent studies across different diagnostic modalities

65 have demonstrated that total CD8 T cell content alone is not a reliable predictor of clinical benefit

66 from ICB (6-10). Part of the reason for this lack of predictive benefit is that a large proportion of

67 CD8 T cells in the tumor microenvironment are passive bystanders that lack tumor reactivity (11,

68 12). Recent elegant work has highlighted that CD8 T cells that express CD39 are enriched for features of a clonal, proliferative lymphocyte population that expresses high levels of activation/exhaustion (e.g. PD-1) and cytotoxicity (e.g. granzyme B) markers (2, 12-15).

71 Moreover, CD39 was highly expressed on empirically defined neoantigen- and tumor-associated

72 antigen-reactive $T$ cells in lung cancer and melanoma $(16,17)$. The largest profiling of CD39

73 expression on CD8 T cells to date in a single disease was performed in 68 patients with head and

74 neck squamous cell cancer; this paper contained an additional 42 samples across a mixture of

75 seven disease types (13). Herein, we describe the tumoral features that are associated with CD39

76 levels on CD8 T cells from 440 clinical samples of human lung cancer, validate the utility of CD39

77 as a surrogate of tumor-reactive CD8 T cells, and report that baseline CD39 on CD8 T cells

78 predicts enhanced efficacy of ICB in patients with lung cancer.

\section{RESULTS}

81 Single-cell cellular indexing of transcriptomes and epitopes (CITE)/RNA/T cell receptor 82 (TCR)seq reveals that CD39 ${ }^{\text {high }}$ CD8 T cells are enriched for features of exhaustion, tumor 83 reactivity, and clonal expansion

84 To characterize CD8 T cells expressing CD39, we sorted CD3 ${ }^{+} \mathrm{T}$ cells from four non-small 85 cell lung cancer (NSCLC) biospecimens and performed droplet-based single-cell 
CITE/RNA/TCRseq (18). The four NSCLC samples comprised a range of histologies (e.g. adenocarcinoma and squamous), driver mutations (KRAS and EGFR), stages (e.g. early and metastatic), and anatomic sites (e.g. lung, pleural fluid, and brain) (Table S1). None of these patients had received prior ICB at the time of sample collection. After coarse clustering, we visualized separated clusters of regulatory T cells, CD4 T cells, CD8 T cells, and an additional T cell NOS (not otherwise specified) cluster (Fig. 1A, Table S2). We focused our subsequent analyses on CD8 $\mathrm{T}$ cells since this subset has the greatest known contribution to anti-tumor immunity. The 896 single CD8 T cells that passed quality control (QC) were divided by CD39 protein expression (assessed by oligo-tagged anti-CD39 antibody) into high (hi), intermediate (int), and negative (neg) groups. Transcriptional dropout is a well-known limitation of single cell RNAseq, and oligonucleotide-tagged antibodies to surface molecules (e.g. CITE-seq) represents a strategy to overcome this hurdle (19). Concordantly, CITEseq detected protein expression of CD39 and other activation markers in many cells in which there was absent transcriptional expression (Fig S1A). Genes that were differentially expressed in CD39hi CD8 T cells included the exhaustion marker $\angle A Y N$, tissue residence marker ITGAE (CD103), and the activation markers CXCL13, GNLY, HLADRA, and VCAM1 (Table S3). CD39hi CD8 T cells had the highest transcriptional levels of ENTPD1 (CD39), PDCD1 (PD-1), ITGAE, CXCL13, TNFRSF4 (OX-40), HAVCR2 (TIM-3), and LAG3, which are features of tumor-reactive CD8 T cells (Fig. S1B) (20). Protein level assessment by CITE-seq revealed that CD39hi CD8 T cells across the four samples consistently expressed the highest protein levels of PD-1, CD103, OX40, 4-1BB, and LAG-3 (Fig. 1B). Concordantly, CD39hi CD8 T cells expressed the highest transcriptomic signature score for T cell exhaustion and tumor reactivity (Fig. 1C-D, Table S4).

Two recent reports demonstrated that empirically validated tumor-reactive CD8 T cells express CD39 $(16,17)$. In these studies, the authors assessed for CD8 tumor reactivity to tumorassociated or viral-associated antigens and generated gene expression signatures of the reactive CD8 T cells. Thus, we next evaluated whether the gene expression profiles of CD39hi CD8 T in

112 our dataset overlapped with the CD8 T cells in these two recent datasets. CD39hi CD8 T cells in 113 our dataset indeed expressed the highest 'tumor specific' and 'MANA-TIL (mutation-associated 114 neoantigen-tumor infiltrating lymphocyte)' signature scores and lowest 'virus specific' and 115 'influenza' signatures (Fig. 1E-F, S1C-D).

Since CD39hi CD8 T cells were enriched for features of tumor reactivity, we hypothesized

117 that these cells would display greater clonal expansion. Indeed CD39hi CD8 T cells expressed the 118 highest proliferation score and both CD39int and CD39hi CD8 T cells comprised a higher clonal 119 proportion among all CD8 T cells (Fig 1G-H). Thus, our single cell CITE/RNA/TCR sequencing 
120

121

122

123

124

125

126

127

128

129

130

131

132

133

134

135

136

137

138

139

140

141

142

143

144

145

146

147

148

149

150

151

152

153

demonstrates that CD39 ${ }^{\text {hi }}$ CD8 T cells express features of exhaustion, tumor reactivity, and clonal expansion.

\section{CD39 expression on CD8 T cells is a non-redundant biomarker}

We compared CD39, Tim-3, 4-1BB, and PD-1 expression by flow cytometry to compare their relative staining resolution, as defined by the separation of the positive and negative population. FACS-based detection of CD39 consistently yielded higher resolution compared to the other three markers (Fig. 2A). The enhanced resolution of CD39 compared to PD-1 is consistent with our prior report that CD39 has more durable expression in CD8 T cells (21). Due to the staining resolution of the CD39 marker and its association with tumor reactivity, we sought to utilize CD39 expression on CD8 T cells to estimate the frequency of tumor-reactive CD8 T cells in lung cancer clinical samples. From August 2018 to September 2021, we evaluated 440 fresh lung cancer clinical specimens by flow cytometry for CD39 expression on CD8 T cells. These biospecimens ranged across stages (I-IV) and lung cancer subtypes (lung adenocarcinoma, squamous cell cancer, and small cell lung cancer) (Table S5). The mean CD8 level was 15.4\% (of $\mathrm{CD} 45^{+}$) and mean CD39 level was $23.3 \%$ (of CD8 T cells), and these were utilized as cutoffs throughout the rest of the study. On univariate analysis, CD39 expression on CD8 T cells had a weak correlation with total CD8 T cells, smoking history, TMB, and PD-L1 (Fig. S2A-D). Since $\mathrm{TMB} \geq 10$ mutations/megabase and PD-L1 $\geq 50 \%$ represent subgroups with favorable clinical outcomes from ICB therapy $(3,4)$, we assessed \%CD39 on these subpopulations. When divided into quartiles by PD-L1 and TMB expression, tumor material from the two TMB $\geq 10$ mutations/megabase subgroups (both PD-L1 $<50 \%$ and $\geq 50 \%$ ) had nearly two-fold higher \%CD39 among CD8 T cells, relative to the TMB $<10$ and PD-L1 $<50 \%$ subgroup (Fig. S2E). In contrast, the proportion of CD8 T cells (Fig. S2F) showed less variation. There was largely no association of CD39 expression on CD8 T cells with lung cancer stage with the exception of a reduction in stage IVA tumors (Fig. S2G). This is concordant with reduced CD39 expression on CD8 T cells in pleural fluid and pleural metastases biospecimens relative to the lung biospecimens (Fig. S2H). Notably, we previously reported that the pleural and peritoneal cavities are immunosuppressed microenvironments due to the presence of $\mathrm{Tim}_{-} 4^{+}$cavity-resident macrophages (21), and this may contribute to the low \%CD39 in these anatomic compartments.

CD39 levels on CD8 $T$ cells showed two patterns of expression among the lung cancer subtypes. ALK fusion, ROS1 fusion, MET exon 14 fusion, BRAF V600E, RET fusion and EGFR mutant (except exon 20 insertion) lung adenocarcinomas, which are not associated with tobacco use, tended to have below average CD39 expression on CD8 T cells (Fig. 2B, Table S6). The 
154 reduced CD39 expression on EGFR mutant lung cancer tumors is in line with a prior report (12).

155 In contrast, CD39 expression on lung adenocarcinomas with KRAS G12C, G12D, G12V or 156 ERBB2 (HER2) mutations were above average. 'Other KRAS' mutations, including G12A, G12R, 157 G12S, G13C, G13D, KRAS Q61H, and Q61R, which are known to be oncogenic per OncoKB 158 (22), were associated with a lower-than-mean CD39 level on CD8 T cells. The proportion of $159 \mathrm{CD} 39+^{+} \mathrm{CD} 8 \mathrm{~T}$ cells in squamous and small cell lung cancers averaged $30.3 \%$ and $27.3 \%$, 160 respectively. In contrast, only $6.4 \%$ of CD8 T cells in neuroendocrine carcinoid (other 161 neuroendocrine) tumors expressed CD39. These findings are consistent with KRAS, squamous, 162 and small-cell lung cancers being strongly associated with tobacco use, which is correlated with 163 a higher number of tumor mutations and neoantigens to which CD8 $T$ cells can react. 164 Nonetheless, even among the lung cancer subtypes with higher \%CD39, there was a wide range of CD39 levels (Fig. 2B), suggesting that even for a given driver mutation, there is substantial heterogeneity in CD8 immunosurveillance. Total CD8 T cell infiltration, TMB, and PD-L1 were overall not associated with consistent differences across lung cancer histological subtypes (Fig. 2C, S2I-J).

Since our data was retrospective and derived from a heterogeneous cohort of patients, we performed multivariate analyses to determine the clinical and molecular features that best correlate with CD39 expression on CD8 T cells. We included tissue site, stage, histology, driver mutation, TMB, smoking history, and PD-L1 as potential covariates. We also included human leukocyte antigen (HLA) heterozygosity $(23,24)$ in the model and also the number of predicted neoantigens and strong binding neoantigens from next-generation sequencing of tumor biopsies by MSK-IMPACT (25). A linear model predicted the variance in \%CD39 on CD8 T cells at an adjusted R-squared of 0.24 , suggesting that the features in the model poorly predict the variance observed in CD39 levels (Table S7). In the model, only TMB, PD-L1, pericardial metastases, and prior chemotherapy passed significance thresholds of $P<0.05$. Since some of the features in the dataset had few observations and to avoid overfitting, we also applied a lasso regression with 10fold cross validation. The lambda was selected based on the root-mean-square error (RMSE) and the model achieved an R-squared of 0.17 with a RMSE of 1.5 at lambda $=0.1$ (Table S8). Notably, at higher lambdas, the algorithm removed all predictors suggesting that there was no linear

184 indicate that CD39 expression on CD8 T cells is a feature of the tumor that is non-redundant to 185 the tumoral parameters that currently guide therapy in lung cancer (e.g. histology, driver 186 alterations PD-L1, and TMB). 


\section{CD39 is upregulated on CD8 T cells in the tumor microenvironment}

We next sought to understand the patterns of tissue-specific expression for CD39 and thus performed CD39 staining on CD8 T cells from matched tumor and peripheral blood. We analyzed three paired samples from peripheral blood and matched tumor lesions with varied anatomic sites (e.g. the brain, lymph node, and lung). In all three cases, high levels of CD39 were only observed in the tumor tissue and not in the peripheral blood (Fig. S3A). This suggests that CD8 T cell interactions with cancer cells drives the expression of CD39 and is in line with our recent report that CD39 was expressed at higher levels in regions of a resected tumor with viable cancer cells compared to regions without viable tumor, normal adjacent regions, and lymph nodes (26). Since there was a small CD39+ population of intermediate intensity in the peripheral blood, we evaluated whether this population gave rise to the CD39+ CD8 T cells in the tumor tissue. To address this question, we sorted CD39- and CD39+ CD8 T cells from the peripheral blood and CD39- or CD39+ from the tumor tissue. We then performed bulk TCR sequencing analysis and assessed for clonal overlap. As assessed by the Morisita Index, there was a minimal level of clonal overlap between CD39+ CD8 T cells from the peripheral blood and CD39+ CD8 T cells from tumor tissue, suggesting that circulating CD39+ CD8 T cells may not be the dominant precursors for the CD39+ CD8 T cells in the tumor microenvironment (Fig. S3A-C).

We have previously shown that CD39 surface expression manifests more durable expression after TCR stimulation compared to other markers associated with activation, such as phosphatidylserine, PD-1, and CD44 (21). Due the greater durability of CD39 even after removal of TCR stimulation, we next assessed the robustness of CD39 levels across spatially and temporally distinct lesions. We analyzed 12 paired samples in which two anatomically distinct lesions were simultaneously assessed (e.g. two resected lung lesions, lymph node and primary lung lesions, or pleural metastasis and fluid). We noted reasonable concordance across spatially distinct lesions (Fig. S3D), as exemplified by the low CD39 level on CD8 T cells from the paired pleural metastasis and fluid from MSK 1266 (Fig. S3E). There was one notable case of divergent

214 CD39 levels. MSK 1372a and b were simultaneous resections of two right-sided separate primary 215 lung cancer lesions with marked differences in CD39 expression (59.0\% vs. 92.5\%; Fig. S3F). 216 Consistent with being separate primary lesions, these two tumors were comprised of two different $217 K R A S$ driver mutations with differential levels of TMB, which may underly the divergence of CD39 218 expression. We also followed CD39 levels on CD8 T cells from the same anatomic site multiple 219 times (e.g. recurrent pleural effusions, initial lung biopsy followed by resection, and serial 220 resections for recurrent disease in the brain). The CD39 levels over time across nine patients 221 were largely durable, including for three brain lesions that were resected over the course of nine 
222 months (MSK 1265; Fig. S3G-H). These findings highlight that CD39 is upregulated on CD8 T

223 cells in proximity to the tumor and that CD39 levels on CD8 T cells are relatively preserved across

224 spatially and temporally distinct lesions in the same patient.

225

\section{EGFR driver mutation-reactive TCRs preferentially express CD39}

A recent report highlighted that CD8 T cell responses to EGFR driver mutations could be detected in the peripheral blood of patients with EGFR-mutant lung cancer and that this could be boosted with neoantigen vaccination (27). Despite poor outcomes with ICB therapy $(28,29)$ and relatively low levels of CD39 on CD8 T cells in patients with EGFR-mutant lung cancers, a population of CD39+ CD8 T cells can be observed in nearly all patient biospecimens from this lung cancer subtype (Fig. 2B). We hypothesized that CD8 T cells reactive to EGFR driver mutations, which would constitute a public neoantigen (30), could be found in EGFR-mutant lung cancer biospecimens and that they would preferentially express CD39 relative to the tetramernegative fraction, which is a mix of tumor-reactive CD8 T cells with other specificities and bystander CD8 T cells. The most common EGFR mutation in lung cancer is a deletion of amino acids 746-750 in exon 19. By utilizing NetMHC 4.0 to evaluate predicted binding of the neopeptide sequence of this mutation to HLA alleles present in biospecimens from patients with EGFR exon 19 deletion mutations in amino acids 746 to 750 , we identified a neopeptide candidate AIKTSPKANK with a $137.83 \mathrm{nM}$ predicted binding affinity to HLA-A*3:01. HLA-A*03:01 is among the most common HLA alleles in North America and Western Europe with a frequency of $20 \%$.

242 We generated an HLA-A*3:01 tetramer loaded with AIKTSPKANK and queried three 243 biospecimens from HLA-A*3:01+ patients with EGFR-mutant lung cancer. We observed tetramer

244 binding above background in two of the three patients (Fig. 3A). In these two patients (MSK 1073a 245 and 1329a), the tetramer-positive CD8 T cells had a higher expression of CD39 compared to the 246 tetramer-negative CD8 T cells (Fig. 3B).

247 Using the same neopeptide binding prediction method above for EGFR exon 21 L858R 248 mutation, which is the second most common EGFR mutation in lung cancer, we predicted binding 249 of the KITDFGRAK neo-peptide to HLA-A*03:01 with $222.48 \mathrm{nM}$ binding affinity. We identified two 250 biospecimens from HLA-A*3:01+ patients with lung cancer harboring an EGFR exon 21 L858R 251 mutation. In both biospecimens, we observed a tetramer-positive population that preferentially 252 expressed CD39 relative to the tetramer-negative CD8 T cells (Fig. 3C-D). These findings 253 collectively demonstrate that CD39 can identify rare neoantigen-reactive CD8 T cells in lung 254 cancer. 


\section{PD-1 axis blockade increases the frequency of CD39+ CD8 T cells}

Since our data and prior reports $(12,16)$ demonstrate that tumor antigen-reactive CD8 T cells express CD39 in lung cancer, we sought to leverage CD39 as a proxy for CD8 anti-tumor immunosurveillance. It has been proposed that chemotherapy can result in 'immunogenic cell death' that can prime an anti-tumor CD8 T cell response (31). We reasoned that CD39 levels would be increased in patients after cytotoxic chemotherapy if such immune priming occurred. Analogously, ICB therapy has been described to expand the pool of antigen-specific CD8 T cells that infiltrate the tumor (32), which we reasoned might be reflected in increased CD39+ CD8 T cells in the tumor. Thus, we sought to evaluate in our dataset whether we found increases of CD39+ CD8 T cells with either cytotoxic chemotherapy or ICB therapy.

We first investigated the 218 patients in our cohort with stage IV lung cancer who were treated with or without chemotherapy and/or ICB in the prior three or six months. Both total CD8 infiltration and CD39 levels on CD8 T cells were unchanged in patients receiving cytotoxic chemotherapy in the preceding three or six months (Fig. 4A-D). Total CD8 infiltration was also unchanged in patients receiving preceding ICB therapy (Fig. 4A,C). In contrast, CD8 T cells from patients that received ICB in the prior three or six months expressed higher levels of CD39 (Fig.

272 4B,D). In patients that received both cytotoxic chemotherapy and ICB therapy in prior three or six

273 months, the level of CD39 on CD8 T cells was unchanged compared to patients that had not 274 received chemotherapy or ICB in the prior 3 or 6 months, which is consistent with chemotherapy and ICB having opposing effects on CD39 expression (Fig. 4B,D).

Since the stage IV lung cancer cohort is quite heterogeneous, we subsequently examined 208 early-stage (stage I-III) NSCLC biospecimens from patients that did or did not receive neoadjuvant therapy prior to sample collection. Among early-stage patients with NSCLC, we again did not observe differences in total CD8 infiltration with preceding neoadjuvant chemotherapy; however, neoadjuvant immunotherapy did increase total CD8 infiltration (Fig. 4E). CD8 T cells from patients who underwent neoadjuvant chemotherapy had reduced CD39 levels compared to patients without neoadjuvant therapy; in contrast, in patients who underwent neoadjuvant ICB, we observed an increase in CD39 expression on CD8 T cells (Fig. 4F). Thus, across two clinical cohorts, we did not find evidence for 'immunogenic cell death' with standard

285 cytotoxic chemotherapy utilized for lung cancer; however, we did discover that ICB exposure was 286 associated with an enhanced infiltration of CD39+ CD8 T cells. This is consistent with recent 287 appreciation that ICB results in clonal expansion and infiltration of tumor-reactive CD8 T cells 288 (32). 


\section{CD39 and PD-1 expression on CD8 T cells portends benefit from ICB therapy}

We next sought to evaluate whether CD39 on CD8 T cells had prognostic significance in our dataset. We first assessed whether CD39 levels at the time of resection in 188 patients with stage I-IIIA lung cancer resulted in differential recurrence-free survival (RFS). Since CD39 levels are modulated by prior ICB (Fig 4F), we excluded patients who had previously received neoadjuvant ICB in this analysis. Across this early-stage resection cohort, we did not observe differences in RFS based on total CD8 T cells or \%CD39 among CD8 T cells utilizing mean cutoff values from the total 440 patient dataset (Fig. 5A-C, Fig. S4A-C). The data for CD8 T cells is in line with a recently published prospective cohort of early-stage NSCLC resection specimens which showed no differences in clinical outcomes based on total CD8 abundance (33). Similarly, neither total CD8 T cells or \%CD39 among CD8 T cells resulted in differential progression-free survival (PFS) in the 26 stage IV lung cancer patients undergoing cytotoxic chemotherapy without ICB (Fig. 5D, S4D). Hence, CD39 expression on CD8 T cells was not prognostic for lung cancer in our dataset.

We next asked whether we could find evidence that CD39 levels on CD8 T cells was associated with response to ICB. There were 22 patients in our cohort from whom we had obtained clinical biospecimens prior to or within three weeks of commencing ICB therapy (Fig. 5E). Although the tumoral PD-L1, TMB, and \%CD8 among DAPI- cells were not different between non-responders (stable disease/progression of disease, SD/POD) and responders (partial response/complete response, PR/CR) to ICB, we observed that responders had a higher level of PD-1 and CD39 on CD8 T cells (Fig. 5F-J). While total CD8 infiltration did not differentiate PFS in these patients (Fig. 5K), patients with higher levels of PD-1 or CD39 on CD8 T cells had an improved PFS ( $p=0.0145$ for PD-1 and $p=0.0063$ for CD39, Fig. 5L-M). Thus, consistent with prior reports in lung cancer $(2,34,35)$, levels of baseline exhausted CD8 T cells are associated with clinical benefit from ICB therapy.

\section{DISCUSSION}

Lung cancer therapy is currently guided by tumor cell-intrinsic features, including histology, driver alterations, PD-L1 and TMB. Integration of tumor cell-extrinsic features such as 319 the immune infiltrate can potentially lead to improved therapeutic options for patients with lung 320 cancer. This was exemplified by the recent report of a 'lung cancer activation module', consisting 321 of activated T cells, plasma cells, and macrophages, that served as a predictor of response to 322 immunotherapy. Since CD8 T cells are the critical effectors in ICB therapy, features of CD8 T 323 cells could also potentially serve as a non-redundant biomarker of response to ICB. Although 
324 methods to empirically verify tumor reactivity have improved substantially in sensitivity and 325 throughput (e.g. MANIFEST (36) and 4-1BB assay (17)), there remains a need in certain contexts 326 for simple and reliable methods to distinguish and quantify the tumor-reactive CD8 sub-population 327 from bystanders without cognate TCR reactivity. We observed from single-cell profiling of 328 heterogeneous lesions that CD8 T cells expressing high levels of CD39 are enriched for features 329 of exhaustion, tumor reactivity and clonal expansion. Motivated by this finding and recent reports 330 that empirically demonstrated that tumor-reactive CD8 T cells express CD39 $(2,12,13,16,17$, 331 20), we profiled CD39 on 440 lung cancer biospecimens obtained at MSKCC. We observed that 332 CD39 on CD8 T cells is expressed more highly in tobacco-associated lung cancer genotypes and 333 only weakly correlates with tumor TMB and PD-L1. Hence, CD39 is a biomarker that is non334 redundant to tumoral features of lung cancer. CD39 is likely dependent on other variables not captured in our dataset, including T cell repertoire, HLA subtype, peptide binding, and likely the intersection of these three highly diverse attributes. CD39 is upregulated on CD8 T cells in the tumor microenvironment relative to the periphery and is generally stable across space and time. Moreover, we demonstrate that rare neoantigen-reactive CD8 T cells preferentially express CD39. This provides additional validation for CD39 serving as a proxy for tumor-reactive CD8 T cells and nominates CD39-guided strategies for TCR-based therapeutics against these targets (37). Finally, we demonstrate that although the level of CD39 on CD8 T cells was not prognostic in any of the patient cohorts that we examined, it was associated with clinical benefit from ICB therapy.

There have been conflicting data regarding whether the presence of CD8 T cells expressing markers of exhaustion can predict clinical benefit from ICB. Single-cell RNAseq of tumors from metastatic melanoma revealed that a CD8 T cell state called CD8_B, which is enriched in ENTPD1 (CD39), PDCD1 (PD-1), CD38, HAVCR2 (TIM-3), and LAG3, is preferentially expressed in non-responders to ICB (9). Also, the use of multiplex immunofluorescence staining in lung cancer suggests that an 'effector burnt out' (EBO) CD8 T effector population preferentially expressing PD-1 and LAG-3 was enriched in patients who did not derive durable benefit from ICB and that these patients had reduced overall survival (38). Notably, CD39 was not a selective feature of the EBO CD8 T cells. On the other hand, lung cancer 352 patients with high pre-treatment levels of PD-1 by flow cytometry or immunofluorescence 353 demonstrated a greater response rate and PFS $(2,34)$. Moreover, high levels of intratumoral 354 CD39+ CD8 T cells is associated with response to ICB therapy in lung cancer (35). These findings 355 dovetail with other studies that revealed that high levels of CD8 T cells with features of exhaustion 356 prior to ICB are associated with improved clinical benefit in colorectal cancer and $\mathrm{ER}^{+}$breast 
cancer $(39,40)$. Our data confirm that the PD-1 and CD39 on CD8 T cells are both predictive of improved outcomes from ICB therapy.

Two recent reports highlight that the predictive significance of CD39 for immunotherapy is context dependent. Caushi et. al. demonstrated that although CD8 T cells that recognize neoantigen mutations express CD39, neoantigen-reactive CD8 T cells that expressed higher

362 levels of CD39 were associated with inferior pathological responses (16). Krishna et. al. similarly

363 showed that neoantigen-reactive CD8 T cells in ex vivo expanded TILs prior to infusion 364 preferentially expressed CD39 and CD69, but improved clinical outcomes were observed in 365 patients whose pre-infusion products harbored a high number of CD39- CD69- TILs (41). One 366 explanation for this apparent paradox may be that the relative timing of the evaluation of 367 exhaustion likely influences the predictive capability of this biomarker since our data demonstrates that CD39 increases after ICB. Notably, in the two cases highlighted above, the correlation of CD39 with worse outcomes was read out after immune based intervention (i.e. ICB or ex vivo expansion followed by adoptive cell transfer). It is plausible that pre-treatment/early on-treatment exhaustion is reflective of a mobilizable pool of tumor-reactive $T$ cells that portends a favorable response to ICB; conversely, exhaustion after a course of ICB might be indicative of a persistent hypofunctional state that is associated with a worse clinical outcome.

There are several limitations of this study that we acknowledge. First, the data are derived from patients treated at a single center. Second, although we further validate that tumor-reactive CD8 T cells express CD39, we have not rigorously assessed the technically challenging converse question: how many of the CD39+ CD8 T cells are tumor-reactive? Third, while we profiled CD39 on CD8 T cells, our data does not capture the likely contribution/modulation of other immune cell types such as B cells, innate lymphoid cells, and myeloid cells. Lastly, our cohort to assess the predictive significance of CD39 expression on CD8 T cells in stage IV lung cancer treated with ICB monotherapy is limited to 22 patients despite $>3$ years of fresh tissue collection. Part of this limited sample size is attributable to the routine incorporation of cytotoxic chemotherapy to ICB for frontline treatment for the majority of patients with lung cancer. Despite these limitations, the data presented here contribute substantially to our understanding of CD39 on CD8 T cells in the context of lung cancer, including factors that modulates its pattern of expression. Our study identifies CD39 on CD8 T cells as a biomarker that is largely independent of TMB and PD-L1,

388 CD8 T cell population. Subsequent studies can also leverage CD39 to enrich for TCR candidates 389 to evaluate for TCR-based immunotherapies. 


\section{ACKNOWLEDGEMENTS}

392 We are grateful for experimental support from the MSKCC Molecular Cytology Core Facility, Flow 393 Cytometry Core Facility, and Integrated Genomics Operation Core (funded by the NCl Cancer 394 Center Support Grant (CCSG, P30 CA08748), Cycle for Survival, and the Marie-Josée and Henry 395 R. Kravis Center for Molecular Oncology. This research was funded in part through the $\mathrm{NIH} \mathrm{NCI}$ 396 CCSG P30 CA008748, NCI R01 CA056821, U24 CA213274, P01 CA129243, R01 CA197936, 397 R37 CA259177 (CAK), Cancer Research Institute CRI3176 (CAK), Stony-Wold Herbert Fund, 398 International Association of Lung Cancer Research (IASLC)/ International Lung Cancer 399 Foundation (ILCF), the MSKCC Society Grant, the Ludwig Collaborative and Swim Across 400 America Laboratory, the Emerald Foundation, the Parker Institute for Cancer Immunotherapy at 401 MSKCC, the Department of Medicine at MSKCC, and Stand Up To Cancer (SU2C)-American 402 Cancer Society Lung Cancer Dream Team Translational research grant (SU2C-AACR-DT17-15). 403 AC was supported by an MSKCC Investigational Cancer Therapeutics Training Program 404 fellowship (T32 CA-009207) and Clinical Investigator Award from National Cancer Institute (K08 405 CA-248723). We are grateful for manuscript editing provided by Dr. Clare Wilhelm and Reeja 406 Thomas.

\section{AUTHOR CONTRIBUTIONS}

$\mathrm{AC}$ conceived the project, performed experiments, analyzed data, drafted the manuscript, and edited the manuscript with assistance from all authors. FZU, AD, NS, VA, PM, ML, AH, MC, MG, and DH performed experiments and analyzed data. LM, HR, ST, SC, AF analyzed data. AQV, JMC, MM, MM, RL, MW, AK, CK, MW, and JY assisted with biospecimen collation and collection. AR, MTAD, GH, CAK, MS, MDH, EdS, and TS supervised experiments and/or analyses. JDW, $\mathrm{TM}$, and CMR conceived and supervised the study and edited the manuscript.

417 CAK received research grant support from Kite/Gilead; is on the Scientific and/or Clinical Advisory 418 Boards of Achilles Therapeutics, Aleta BioTherapeutics, Bellicum Pharmaceuticals, Catamaran 419 Bio, Obsidian Therapeutics, and T-knife, and has performed consulting services for Bristol Myers 420 Squibb, PACT Pharma, and Roche/Genentech. CAK is a co-inventor on patent applications 421 related to TCRs targeting public neoantigens unrelated to the current work. MDH received 422 research grant from BMS; personal fees from Achilles, Arcus, AstraZeneca, Blueprint, BMS, 423 Genentech/Roche, Genzyme, Immunai, Instil Bio, Janssen, Merck, Mirati, Natera, Nektar, Pact 424 Pharma, Regeneron, Shattuck Labs, Syndax, as well as equity options from Arcus, Factorial, 
425 Immunai, and Shattuck Labs. A patent filed by MSKCC related to the use of tumor mutational

426 burden to predict response to immunotherapy (PCT/US2015/062208) is pending and licensed by

427 PGDx. JDW is a consultant for Adaptive Biotech, Amgen, Apricity, Ascentage Pharma, Arsenal

$428 \mathrm{IO}$, Astellas, AstraZeneca, Bayer, Beigene, Boehringer Ingelheim, Bristol Myers Squibb, Celgene,

429 Chugai, Daiichi Sankyo, Dragonfly, Eli Lilly, Elucida, F Star, Georgiamune, Idera, Imvaq, Kyowa

430 Hakko Kirin, Linneaus, Maverick Therapeutics, Merck, Neon Therapeutics, Polynoma, Psioxus,

431 Recepta, Takara Bio, Trieza, Truvax, Trishula, Sellas, Serametrix, Surface Oncology, Syndax,

432 Syntalogic, and Werewolf Therapeutics. JDW has received grant/research support from Bristol

433 Myers Squibb and Sephora. JDW has equity in Tizona Pharmaceuticals, Adaptive

434 Biotechnologies, Imvaq, Beigene, Linneaus, Apricity, Arsenal IO, and Georgiamune. JDW is a co-

435 inventor on patent applications related to heteroclitic cancer vaccines and recombinant poxviruses

436 for cancer immunotherapy. JDW and TM are co-inventors on patent applications related to CD40

437 and in situ vaccination (PCT/US2016/045970). TM is a consultant for Immunos Therapeutics and

438 Pfizer. TM is a cofounder of and equity holder in IMVAQ Therapeutics. TM receives research

439 funding from Bristol-Myers Squibb, Surface Oncology, Kyn Therapeutics, Infinity

440 Pharmaceuticals, Peregrine Pharmaceuticals, Adaptive Biotechnologies, Leap Therapeutics, and

441 Aprea Therapeutics. TM is an inventor on patent applications related to work on oncolytic viral

442 therapy, alpha virus-based vaccine, neoantigen modeling, CD40, GITR, OX40, PD-1, and CTLA-

443 4. C.M.R. has consulted regarding oncology drug development with AbbVie, Amgen, Ascentage,

444 AstraZeneca, BMS, Celgene, Daiichi Sankyo, Genentech/Roche, Ipsen, Loxo and PharmaMar

445 and is on the scientific advisory boards of Elucida, Bridge and Harpoon.

447 FIGURE LEGENDS

448 Figure 1. Single-cell CITE/RNA/TCR-sequencing reveals that CD39hi CD8 T cells are 449 enriched for features of exhaustion, tumor-reactivity, and clonal proliferation in human

450 lung cancer. A) UMAP of sorted $\mathrm{CD}^{+} \mathrm{T}$ cells from four patients with lung cancer (see Table S1

451 for patient characteristics). Clusters are annotated on left panel. Surface levels of CD4, CD8, and

452 CD39 as assessed by CITEseq antibody-derived tags (adt) are depicted in right three panels. B)

453 Levels of various proteins (each column) across the four samples (each row) as determined by

454 CITEseq adt levels. C-G) Scaled scores for exhaustion, tumor reactivity, tumor specific, virus

455 specific, and proliferation gene signatures (see Table S4 for full gene list). H) Clonal proportion

456 among CD8 T cells of clonotypes that were categorized by mean CD39 expression. Statistical

457 significance was determined with two-way ANOVA with Tukey's multiple comparisons test and $p$

458 value is indicated if $<0.05$. 
Figure 2. CD39 expression among CD8 TILs varies with lung cancer subtype. A) Representative flow cytometry staining of CD39, Tim-3, 4-1BB, and PD-1 on DAPI- CD45+ ${ }^{+}$C $^{+}$ $\mathrm{CD}^{+} \mathrm{T}$ cells from MSK 1105b. Left plot represents fluorescence minus one (FMO) and right plot represents the CD8 T cells stained with the indicated antibody. B-C) Violin plots of \%CD39+ (among CD8 ${ }^{+}$T cells) and \%CD8 (among CD45+) for various histological subtypes/driver mutation categories among the 440-sample cohort. Dashed line indicates 23.3\% mean CD39 level for entire 440-sample cohort. Red bars indicate histological subtypes/driver mutations with above average \%CD39 values.

Figure 3. Tumor neoantigen-specific CD8 T cells express CD39. A) Flow cytometry plots from three patients with EGFR ex19 p.E746_A750del mutant lung cancer showing staining for A3:01: AIKTSPKANK tetramer. Top row depicts staining of A3:01: AIKTSPKANK tetramer negative control. Bottom row depicts staining of A3:01: AIKTSPKANK tetramer. B) Flow cytometry histogram showing \%CD39+ among tetramer (Tet) $^{-}$and tetramer ${ }^{+}$(Tet $^{+}$) CD8 T cells. C) Flow cytometry plots from two patients with EGFR exon 21 L858R mutant lung cancer staining for A3:01: KITDFGRAK tetramer. Top row depicts staining of A3:01: KITDFGRAK tetramer negative control. Bottom row depicts staining of A3:01: KITDFGRAK tetramer. D) Flow cytometry histogram showing \%CD39+ among tetramer ${ }^{-}$(Tet $\left.^{-}\right)$and tetramer ${ }^{+}\left(\right.$Tet $\left.^{+}\right)$CD8 T cells.

Figure 4. PD-1 axis blockade increases the frequency of CD39+ CD8 tumor-infiltrating lymphocytes.

A-B) \%CD8 (among CD45+) or \%CD39 (among CD8 T cells) from 218 biospecimens obtained from patients with stage IV metastatic disease that did or did not receive chemotherapy or ICB therapy in the prior 3 months. Statistical significance was assessed by two-tailed student's t-test. C-D) \%CD8 (among CD45+) or \%CD39 (among CD8 T cells) from 218 biospecimens obtained from patients with stage IV metastatic disease that did or did not receive chemotherapy or ICB therapy in the prior 6 months. Statistical significance assessed by two-tailed student's t-test. E-F)

$487 \%$ CD8 (among CD45+) or \%CD39 (among CD8 T cells) from 208 biospecimens obtained from 488 patients with early stage (stage I-III) NSCLC. Statistical significance was assessed by two-tailed 489 student's t-test. ${ }^{*} \mathrm{p}<0.05,{ }^{* *} \mathrm{p}<0.001$. 
493 Kaplan-Meier curve of recurrence-free survival after resection with above or below mean \%CD39

494 (among CD8 T cells) for stage I, stage II, and stage IIIA lung cancer patients who did not receive 495 ICB. Statistical significance determined by Mantel-Cox test. D) Kaplan-Meier curve of 496 progression-free survival after resection with above or below mean \%CD39 (among CD8 T cells)

497 for stage IV lung cancer patients who did not receive ICB. Statistical significance determined by 498 Mantel-Cox test. E) Cohort of stage IV lung cancer patients who received ICB monotherapy. F-J) 499 Tumor proportion score for PD-L1, tumor mutation burden, \%CD8 (among CD45+), PD-1 mean 500 fluorescence intensity (MFI) and \% CD39 (among CD8 T cells) among non-responders (stable 501 disease/progression of disease, SD/POD) or responders (partial response/complete response, $502 \mathrm{PR} / \mathrm{CR}$ ) to ICB in the cohort described in E). K-M) Kaplan-Meier survival curve of progression503 free survival patients described in cohort E based on stratification for CD8, PD-1, and CD39. 504 Statistical significance was determined by Mantel-Cox test.

505

\section{STAR METHODS}

\section{Human biospecimens}

508 Fresh primary tumors, metastatic lesions and pleural/peritoneal/pericardial effusions were 509 obtained from August 2018 to September 2021 with permission from the MSKCC IRB. Informed consent was collected from all patients enrolled in this study. Clinical samples were annotated 511 with tumor histology and driver mutation. Adenocarcinoma, adenosquamous, and NSCLC NOS 512 tumors were annotated by their molecular driver mutations, if known. The category 'Unknown 513 Driver' refers to adenocarcinoma, adenosquamous, and NSCLC NOS histology tumors for which 514 a driver mutation (defined as 'known to be oncogenic' by OncoKB (22)) was not identified; notably, 515 this category does not include squamous or small-cell histology biospecimens. With the exception 516 of a single case in which a tumor sample with squamous histology harbored a MET exon 14 517 mutation and two transformed small-cell lung cancer tumors with EGFR mutations, squamous 518 and small-cell lung cancers were annotated by their histology.

\section{Flow cytometry}

521 Cells were incubated with TruFCX (Biolegend) to block nonspecific binding, and then stained $522\left(15 \mathrm{~min}, 4^{\circ} \mathrm{C}\right)$ with appropriate dilutions of various combinations of fluorochrome-conjugated anti523 mouse antibodies. The stained cells were acquired on a LSRII Flow Cytometer or Aria 7 cell sorter 524 and the data were processed using FlowJo software (Treestar). Doublets and dead cells were 525 excluded based on forward scatter (FSC) and side scatter (SSC) and 4',6-diamidino-2526 phenylindole staining (DAPI, $1 \mu \mathrm{g} / \mathrm{ml}$; ThermoFisher). All depicted flow cytometry plots were pre- 
527 gated on non-debris (by FSC and SSC), viable (DAPI-) single CD45 ${ }^{+} \mathrm{CD}^{+}$cells, unless otherwise

528 indicated in the Figure legend. Gating for \%CD39 was determined by gating of fluorescence minus

529 one (FMO). PD-1 mean fluorescence intensity was measured for a subset of samples.

\section{Single-cell transcriptome sequencing}

532 Sorted or dissociated tumor cells were stained with Trypan blue and Countess II Automated Cell 533 Counter (ThermoFisher) was used to assess both cell number and viability. Following QC, the 534 single-cell suspension was loaded onto Chromium Chip A or Next GEM Chip K (10X Genomics 535 PN 230027/1000286) and GEM generation, cDNA synthesis, cDNA amplification, and library 536 preparation of 700-3,300 cells proceeded using the Chromium Single Cell 5' Reagent Kit or Next 537 GEM Single Cell 5' Kit v2 (10X Genomics PN 1000006/1000263) according to the manufacturer's 538 protocol. cDNA amplification included 14-16 cycles and $5.8 \mathrm{ng}-20 \mathrm{ng}$ of the material was used to prepare sequencing libraries with 16 cycles of PCR. Indexed libraries were pooled equimolar and sequenced on a NovaSeq 6000 in a PE26/91 or PE28/91 run using the NovaSeq 6000 SP or S1 Reagent Kit (100 cycles) (Illumina). An average of 108 million reads were generated per sample.

\section{Single-cell V(D)J analysis from RNA}

544 An aliquot of complementary DNA (cDNA) generated using the methods described above was used to enrich for $\mathrm{V}(\mathrm{D}) \mathrm{J}$ regions using the Chromium Single Cell V(D)J Enrichment Kit Human $\mathrm{T}$ Cell (10X Genomics PN 1000005) according to the manufacturer's protocol with 10 cycles of PCR

547 during enrichment and 9 cycles during library preparation. Indexed libraries were pooled 548 equimolar and sequenced on a NovaSeq 6000 in a PE150 or PE26/91 run using the NovaSeq 5496000 SP or S4 Reagent Kit (100 or 300 cycles) (Illumina). An average of 24 million paired reads 550 was generated per sample.

\section{Cell surface protein feature barcode analysis}

553 Amplification products generated using the methods described above included both cDNA and

554 feature barcodes tagged with cell barcodes and unique molecular identifiers. Smaller feature 555 barcode fragments were separated from longer amplified cDNA using a 0.6X cleanup with 556 aMPure XP beads (Beckman Coulter catalog \# A63882). Libraries were constructed using the 557 Chromium Single Cell 5' Feature Barcode Library Kit (10X Genomics PN 1000080) according to 558 the manufacturer's protocol with 9 cycles of PCR. Indexed libraries were pooled equimolar and 559 sequenced on a NovaSeq 6000 in a PE26/91 or PE28/91 run using the NovaSeq 6000 SP or S2 
Reagent Kit (100 cycles) (Illumina). An average of 60 million paired reads was generated per sample.

\section{Single cell CITE/RNA/TCR analysis}

564 Single-cell sequencing data were aligned to the Genome Reference Consortium Human Build 38 565 (GRCh38) using Cell Ranger (v3.1.0; 10X Genomics) in order to obtain T cell clonotypes, feature 566 barcoding, CITEseq antibody detection and gene expression profiles associated with individual 567 single cells. Each data type was matched to create a UMI matrix and cells were filtered out based 568 on three metrics: (1) cells with fewer than 200 detectable genes; (2) cells with more than 3000 569 detectable genes; (3) cells that had fewer than 5\% percentage of counts related to mitochondrial 570 genes. Data normalization, Principal Component Analysis and subsequent Uniform Manifold 571 Approximation and Projections (UMAP) were performed on the dataset using the $\mathrm{R}$ package 572 Seurat v.3.1.1 (https://github.com/satijalab/seurat). The differential expression comparisons were generated using the DESeq2 package with selected genes (FDR < 0.05). After filtering, we created subclusters of cells using the 26 Louvain algorithm. Raw counts were normalized by library size per cell. CD39 ${ }^{\text {neg }}$ was defined as a normalized value of 0 for adt_CD39. CD39 int was defined as the adt_CD39 level between 0 and 1.0 (non-inclusive) since 1.0 was the mean of all non-zero values for adt_CD39 among all the cells in the CD8 cluster. CD39hi was defined as adt_CD39 level greater than or equal to 1.0. Signature scores for exhaustion and T cell reactivity were calculated by AddModuleScore in Seurat. Clonal proportion was calculated as the fractional representation of all CD8 clones by clonotypes that were categorized by mean adt_CD39 expression with the same cutoffs for CD39low, CD39int, and CD39hi as above.

\section{Multivariate analyses}

584 We used the R package caret (42) to implement the glmnet (43) algorithm and evaluate the 585 performance of a lasso regression model using 10-fold cross-validation. The data was center 586 and scaled during preprocessing and lambda was chosen based on the minimal RMSE 587 value. Additionally a linear model was evaluated using the R function "Im". In both models, the 588 response variable, CD39 expression, was log base 2 transformed as well as the predictor 589 variables TMB and total neoantigens per sample.

\section{Tetramer staining}

592 Predicted peptide binding was evaluated by NetMHC version 4.0. The neopeptides derived from 593 the EGFR ex19 p.E746_A750del (AIKTSPKANK) and EGFR ex21 p.L858R (KITDFGRAK) were 
594

595

596

597

598

599

600

601

602

603

604

605

606

607

608

609

610

611

612

613

614

615

616

617

618

619

620

621

622

623

624

625

626

627

ordered from Genscript, diluted to $10 \mathrm{mg} / \mathrm{ml}$ in DMSO and stored in aliquots at $-20^{\circ} \mathrm{C}$. Flex-T MHC Monomers of HLA-A*03:01 were ordered from Biolegend. The UV-labile peptide was exchanged for tested peptide and then conjugated to Streptavidin-PE according to the manufacturer's protocol. The negative control was loaded monomers not conjugated to Streptavidin-PE.

\section{Clinical outcomes analyses}

RFS assessment was performed on 188 biospecimens obtained from stage I-IIIA lung cancer who did not receive neoadjuvant ICB and were not lost to follow up after resection. For the PFS assessment in stage IV patients not treated with ICB, only patients that received at least two cycles of platinum-based chemotherapy were included in the analysis $(n=26)$. For the PFS assessment in stage IV patient treated with ICB, only patients that received at least two cycles of ICB without chemotherapy were included in the analysis $(n=22)$. The cutoffs for \%CD8 and \%CD39 of 15.4 and 23.3 were selected from the means of the 440-sample cohort. The PD-1 cutoff was selected to divide the cohort above and below the median for the stage IV cohort. Response criteria were annotated per RECIST v1.1.

\section{Statistical analysis}

Data are expressed and statistical analyses were performed as described in the Figure legend for each analysis. Statistical significance was determined by two-way ANOVA with Tukey's multiple comparison test, student's t-test or Mantel-Cox log-rank test using Prism 7 software as indicated.

\section{Materials availability}

This study did not generate new unique reagents.

\section{Data availability}

All data generated and supporting the findings of this study are available within the paper. The single-cell CITE/TCR/RNAseq will be deposited in the Gene Expression Omnibus (GEO) database. The TCRseq data will be available through ImmuneACCESS on the Adaptive Biotechnologies website. 


\section{SUPPLEMENTAL INFORMATION}

629 Figure S1. Single-cell CITE/TCR/RNA-sequencing reveals that CD39 ${ }^{\text {hi }}$ CD8 T cells are 630 enriched for features of tumor, but not viral, reactivity.

631 A) Correlation of transcriptional expression ( $y$ axis) with protein expression ( $x$ axis) for indicated 632 genes. B) Pooled transcript levels (scaled counts) of indicated genes in CD39hi, CD39int, and 633 CD39 ${ }^{\text {neg }}$ CD8 T cells as classified by adt_CD39 levels. C-D) Scaled scores for MANA-TIL and 634 influenza- reactivity gene signatures (see Table S4 for full gene list). Statistical significance was 635 determined by two-way ANOVA with Tukey's multiple comparisons test and $p$ value is indicated 636 if $<0.05$.

Figure S2. Clinical and molecular correlates of CD39 expression on CD8 tumor-infiltrating lymphocytes. A-D) Correlation of \%CD39 on CD8 T cells with \%CD8 (among CD45 ${ }^{+}$), tumor mutation burden, PD-L1 tumor proportion score, and smoking history. E-F) \%CD39+ (among CD8 T cells) and \%CD8 (among CD45+) on four subgroups based on cutoffs for TMB and PDL-1 in the 440-sample cohort. Statistical significance was assessed by two-tailed student's t-test. G) Correlation of \%CD39 on CD8 T by lung cancer stage. H) Correlation of \%CD39 on CD8 T by anatomic site of biospecimen. I-J) Correlation of tumor mutation burden and PD-L1 tumor proportion score with various histological subtypes/driver mutation categories among 440 -sample cohort. Red bars indicate histological subtypes/driver mutations with above mean \%CD39 values.

Figure S3. CD39 is upregulated in the tumor microenvironment and is consistent across space and time. A-C) Top row depicts flow cytometry histogram plots of CD39 levels on DAPI' $\mathrm{CD}_{4}{ }^{+} \mathrm{CD}^{+} \mathrm{CD}^{+} \mathrm{T}$ cells from peripheral blood or matched tumor lesion from three patients with lung cancer. Bottom row depicts Morisita indices depicting TCR- $\beta$ overlap among sorted CD39-

652 and CD39+ fractions of blood and tumor CD8 T cells. D) \%CD39 among CD8 T cells from two 653 spatially distinct lesions that were sampled concurrently in the same patient $(n=12)$. Blue line 654 indicates MSK 1266 whose CD39 expression is depicted in E. Purple line indicates MSK 1372 655 depicted in F. E) Flow cytometry plots of CD39 expression on CD8 T cells on two concurrent 656 pleural lesions in MSK 1266. F) Flow cytometry plots of CD39 expression on CD8 T cells in two 657 concurrent spatially distinct lung lesions in MSK 1372. G) \%CD39 among CD8 T cells from two 658 lesions from the same anatomic site that were sampled at temporally distinct times in the same 659 patient $(n=9)$. Orange line indicates MSK 1265 whose CD39 expression is depicted in $H$. H) Flow 660 cytometry plots of CD39 expression on CD8 T cells over the course of three brain resection 661 lesions in MSK 1265. 
662 Figure S4. Total CD8 tumor-infiltrating lymphocytes is not prognostic for survival in lung

663 cancer. A-C) Kaplan-Meier curve of recurrence-free survival stratified by \%CD8 (among CD45 ${ }^{+}$)

664 for stage I, stage II, and stage IIIA lung cancer patients who did not receive ICB. Statistical

665 significance was determined by Mantel-Cox test. D) Kaplan-Meier curve of progression-free

666 survival stratified by \%CD8 (among CD45 $)$ for stage IV lung cancer patients who did not receive

667 ICB. Statistical significance was determined by Mantel-Cox test.

668

669 Table S1. Clinical features of four lung cancer clinical biospecimens that underwent single cell

670 CITE/TCR/RNA sequencing.

671 Table S2. Differentially expressed genes for CD8, CD4, T cell NOS, and Treg clusters from single

672 cell CITE/TCR/RNA sequencing.

673 Table S3. Differentially expressed genes for CD39hi CD8 T cells compared to all other CD8 T

674 cells from single cell CITE/TCR/RNA sequencing.

675 Table S4. List of gene signatures used for scoring.

676 Table S5. Clinical and molecular features of 440 patient lung cancer cohort.

677 Table S6. Median and mean values for \%CD39 on CD8 T cells among lung cancer subtypes.

678 Table S7. Linear regression multivariate analysis of \%CD39 on CD8 T cells.

679 Table S8. Lasso regression multivariate analysis of \%CD39 on CD8 T cells. 


\section{REFERENCES}

683 1. A. Ribas, J. D. Wolchok, Cancer immunotherapy using checkpoint blockade. Science 359, 1350-1355 (2018).

3. M. D. Hellmann et al., Nivolumab plus Ipilimumab in Lung Cancer with a High Tumor

4. M. Reck et al., Pembrolizumab versus Chemotherapy for PD-L1-Positive Non-Small-Cell Lung Cancer. N Engl J Med 375, 1823-1833 (2016).

6. N. Riaz et al., Tumor and Microenvironment Evolution during Immunotherapy with

7. V. Verma et al., PD-1 blockade in subprimed CD8 cells induces dysfunctional PD-

8. B. A. Helmink et al., B cells and tertiary lymphoid structures promote immunotherapy response. Nature 577, 549-555 (2020).

9. M. Sade-Feldman et al., Defining T Cell States Associated with Response to Checkpoint Immunotherapy in Melanoma. Cell 175, 998-1013 e1020 (2018).

10. J. C. Osorio et al., Lesion-Level Response Dynamics to Programmed Cell Death Protein (PD-1) Blockade. J Clin Oncol 37, 3546-3555 (2019).

11. W. Scheper et al., Low and variable tumor reactivity of the intratumoral TCR repertoire in human cancers. Nat Med 25, 89-94 (2019).

12. Y. Simoni et al., Bystander CD8(+) T cells are abundant and phenotypically distinct in human tumour infiltrates. Nature 557, 575-579 (2018).

13. T. Duhen et al., Co-expression of CD39 and CD103 identifies tumor-reactive CD8 T cells in human solid tumors. Nat Commun 9, 2724 (2018).

\section{H. Li et al., Dysfunctional CD8 T Cells Form a Proliferative, Dynamically Regulated} Compartment within Human Melanoma. Cell, (2018).

15. K. E. Yost et al., Clonal replacement of tumor-specific T cells following PD-1 blockade. Nat Med 25, 1251-1259 (2019).

16. J. X. Caushi et al., Transcriptional programs of neoantigen-specific TIL in anti-PD-1treated lung cancers. Nature, (2021). 
716 17. G. Oliveira et al., Phenotype, specificity and avidity of antitumour CD8(+) T cells in 717 melanoma. Nature 596, 119-125 (2021).

718 18. M. Stoeckius et al., Simultaneous epitope and transcriptome measurement in single cells. Nat Methods 14, 865-868 (2017).

19. S. H. Gohil, J. B. lorgulescu, D. A. Braun, D. B. Keskin, K. J. Livak, Applying highdimensional single-cell technologies to the analysis of cancer immunotherapy. Nat Rev Clin Oncol 18, 244-256 (2021).

20. A. M. van der Leun, D. S. Thommen, T. N. Schumacher, CD8(+) T cell states in human cancer: insights from single-cell analysis. Nat Rev Cancer 20, 218-232 (2020).

21. A. Chow et al., Tim-4(+) cavity-resident macrophages impair anti-tumor CD8(+) T cell immunity. Cancer Cell 39, 973-988 e979 (2021).

22. D. Chakravarty et al., OncoKB: A Precision Oncology Knowledge Base. JCO Precis Oncol 2017, (2017).

23. D. Chowell et al., Evolutionary divergence of HLA class I genotype impacts efficacy of cancer immunotherapy. Nat Med 25, 1715-1720 (2019).

24. D. Chowell et al., Patient HLA class I genotype influences cancer response to checkpoint blockade immunotherapy. Science 359, 582-587 (2018).

25. A. Zehir et al., Mutational landscape of metastatic cancer revealed from prospective clinical sequencing of 10,000 patients. Nat Med 23, 703-713 (2017).

26. J. A. Pai et al., Regional and clonal T cell dynamics at single cell resolution in immune checkpoint blockade. bioRxiv, 2021.2009.2027.461389 (2021).

27. F. Li et al., Neoantigen vaccination induces clinical and immunologic responses in nonsmall cell lung cancer patients harboring EGFR mutations. J Immunother Cancer 9, (2021).

28. J. F. Gainor et al., EGFR Mutations and ALK Rearrangements Are Associated with Low Response Rates to PD-1 Pathway Blockade in Non-Small Cell Lung Cancer: A Retrospective Analysis. Clin Cancer Res 22, 4585-4593 (2016).

746 30. C. A. Klebanoff, J. D. Wolchok, Shared cancer neoantigens: Making private matters $747 \quad$ public. J Exp Med 215, 5-7 (2018).

748 31. L. Galluzzi, A. Buque, O. Kepp, L. Zitvogel, G. Kroemer, Immunogenic cell death in cancer and infectious disease. Nat Rev Immunol 17, 97-111 (2017). 
32. K. E. Yost, H. Y. Chang, A. T. Satpathy, Recruiting T cells in cancer immunotherapy. Science 372, 130-131 (2021).

752 33. L. Federico et al., Distinct tumor-infiltrating lymphocyte landscapes are associated with clinical outcomes in localized non-small cell lung cancer. Ann Oncol, (2021).

34. S. Kumagai et al., The PD-1 expression balance between effector and regulatory T cells predicts the clinical efficacy of PD-1 blockade therapies. Nat Immunol 21, 1346-1358 (2020).

35. J. Yeong et al., Intratumoral CD39(+)CD8(+) T Cells Predict Response to Programmed Cell Death Protein-1 or Programmed Death Ligand-1 Blockade in Patients With NSCLC. J Thorac Oncol, (2021).

36. L. Danilova et al., The Mutation-Associated Neoantigen Functional Expansion of Specific T Cells (MANAFEST) Assay: A Sensitive Platform for Monitoring Antitumor Immunity.

37. S. S. Chandran, C. A. Klebanoff, T cell receptor-based cancer immunotherapy:

765

38. M. F. Sanmamed et al., A burned-out CD8+ T-cell subset expands in the tumor

39. M. Chalabi et al., Neoadjuvant immunotherapy leads to pathological responses in MMRproficient and MMR-deficient early-stage colon cancers. Nat Med 26, 566-576 (2020).

769

40. M. Terranova-Barberio et al., Exhausted T cell signature predicts immunotherapy response in ER-positive breast cancer. Nat Commun 11, 3584 (2020).

771 41. S. Krishna et al., Stem-like CD8 T cells mediate response of adoptive cell immunotherapy against human cancer. Science 370, 1328-1334 (2020).

773 42. M. Kuhn, Building Predictive Models in R Using the caret Package. Journal of Statistical 774 Software 28, 1 - 26 (2008).

775 43. J. Friedman, T. Hastie, R. Tibshirani, Regularization Paths for Generalized Linear Models via Coordinate Descent. J Stat Softw 33, 1-22 (2010). 

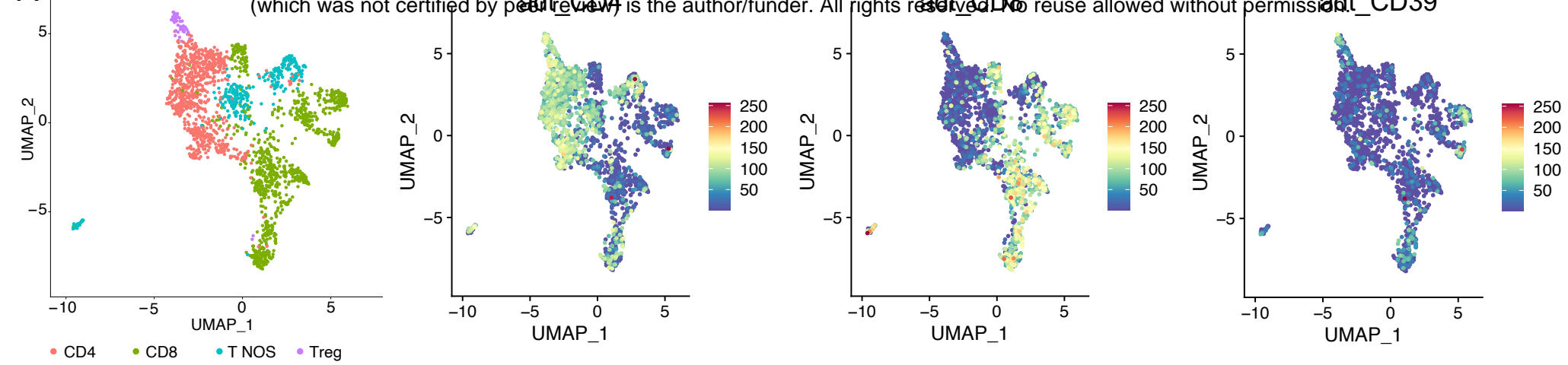

B Protein

PD-1

CD103

OX-40

4-1BB

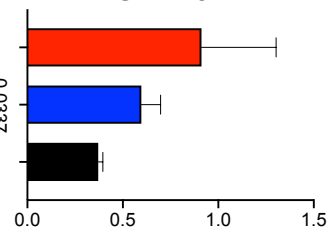

LAG-3

$1087 a$

KRAS

$\mathrm{G} 12 \mathrm{C}$
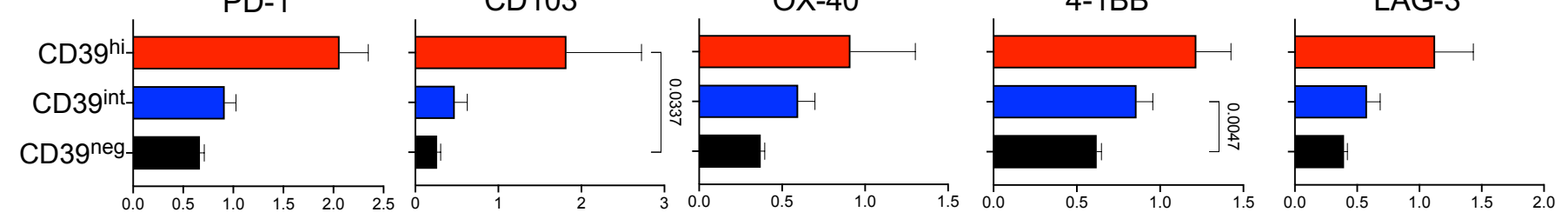

1111a

EGFR

ex19 del
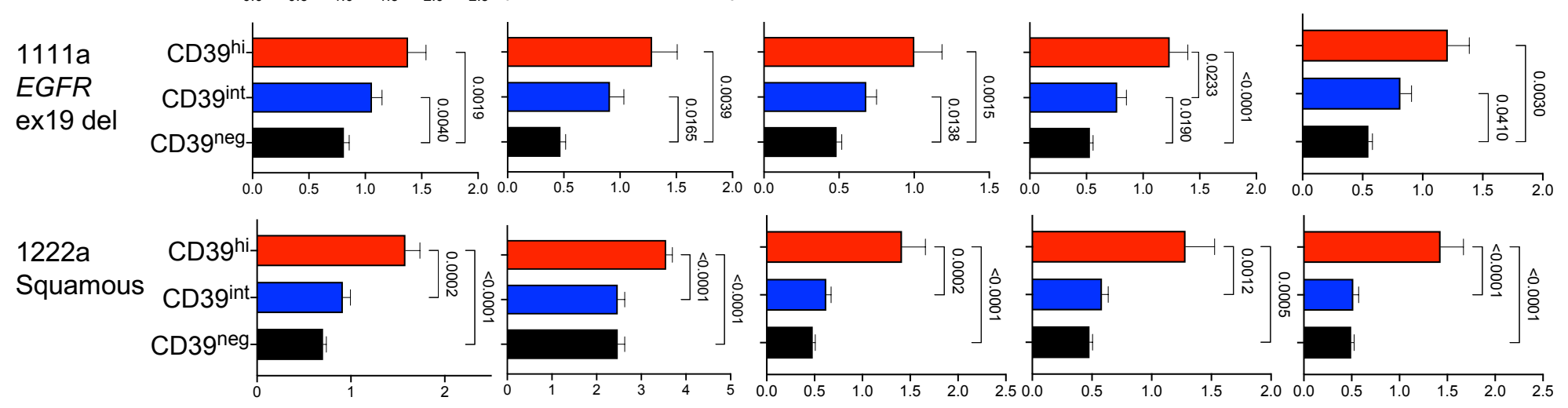

$1256 a$

EGFR

ex19 del
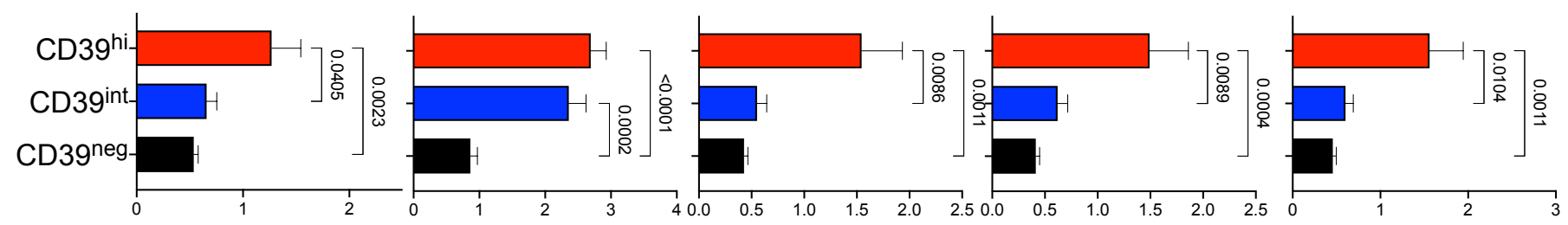

C

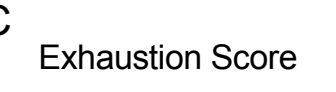

D van der Leun et al
Tumor Reactivity Score

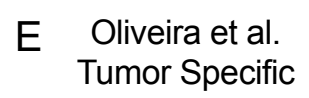

F Oliveira et al.

G

Proliferation Score

H Clonal Proportion

0
0
0
0
0

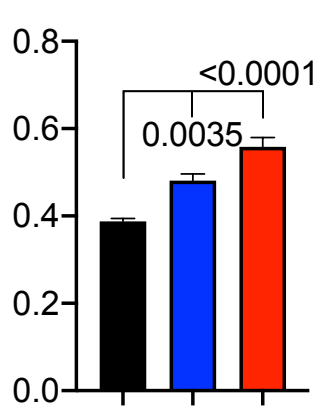

c
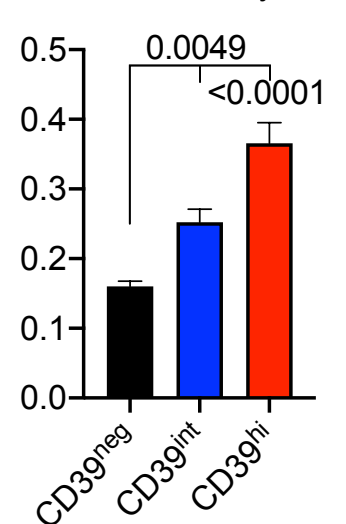
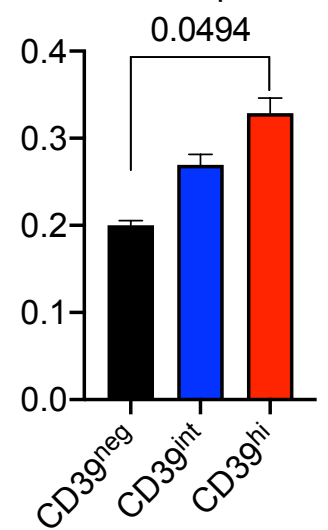
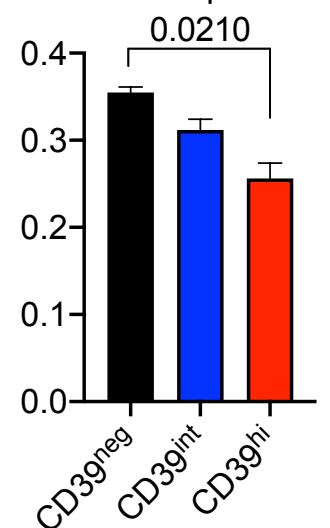
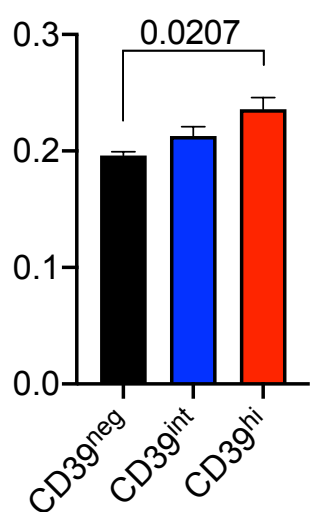

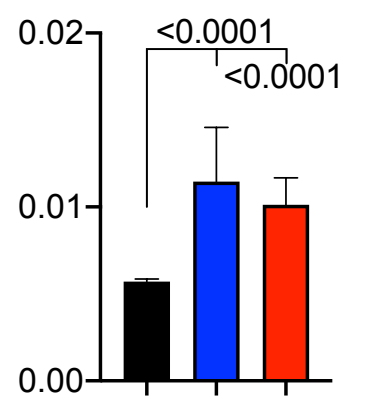

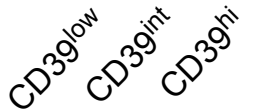

Figure 1. Single-cell CITE/RNA/TCR-sequencing reveals that CD39 hi CD8 T cells are enriched for features of exhaustion, tumor-reactivity, and clonal proliferation in human lung cancer. A) UMAP of sorted CD3 ${ }^{+} \mathrm{T}$ cells from four patients with lung cancer (see Table S1 for patient characteristics). Clusters are annotated on left panel. Surface levels of CD4, CD8, and CD39 as assessed by CITEseq antibody-derived tags (adt) are depicted in right three panels. B) Levels of various proteins (each column) across the four samples (each row) as determined by CITEseq adt levels. C-G) Scaled scores for exhaustion, tumor reactivity, tumor specific, virus specific, and proliferation gene signatures (see Table S4 for full gene list). H) Clonal proportion among CD8 T cells of clonotypes that were categorized by mean CD39 expression. Statistical significance was determined with two-way ANOVA with Tukey's multiple comparisons test and $p$ value is indicated if $<0.05$. 


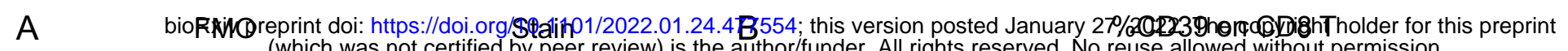

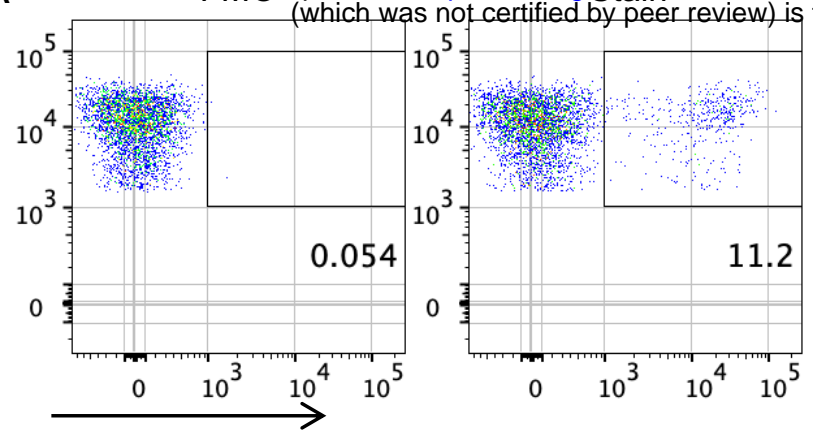

CD39-APC
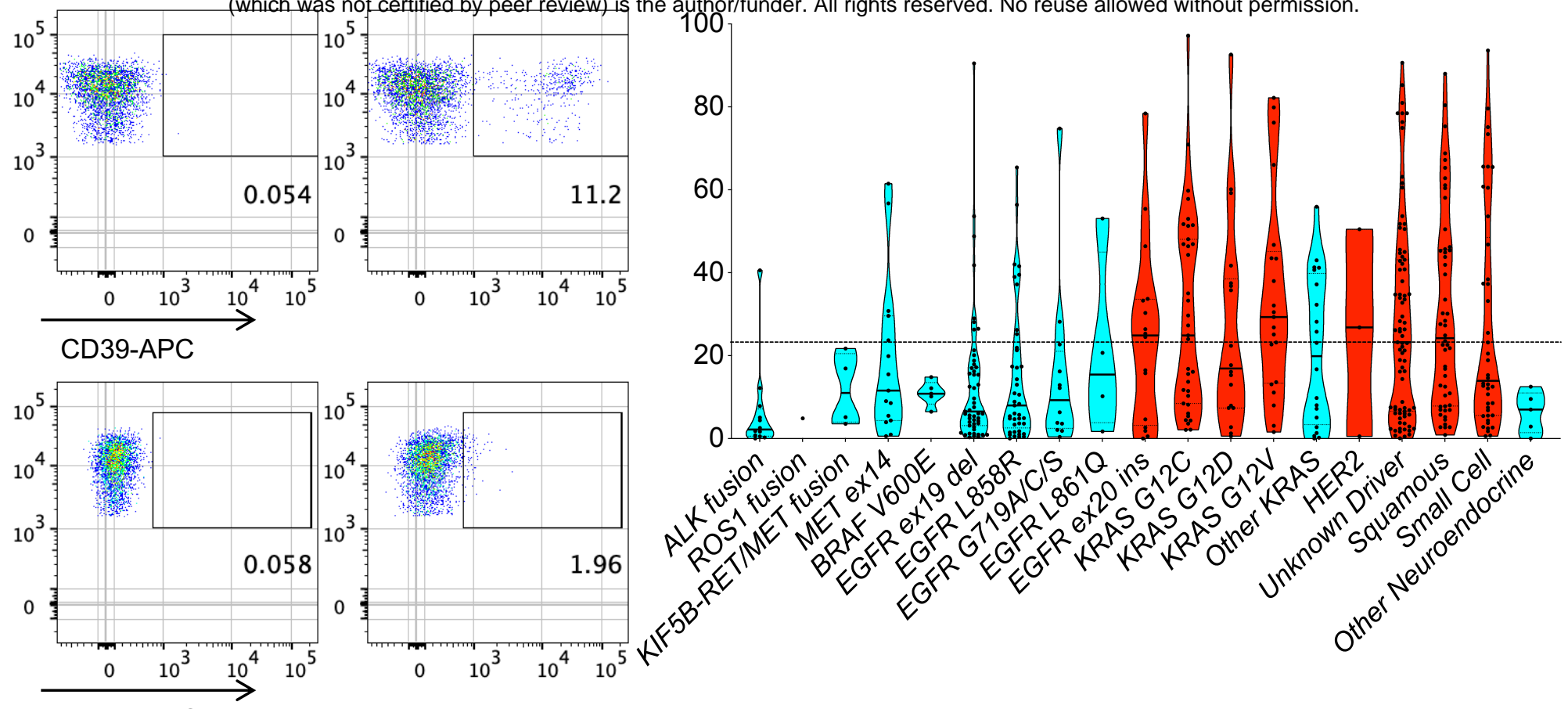

Tim-3-FITC
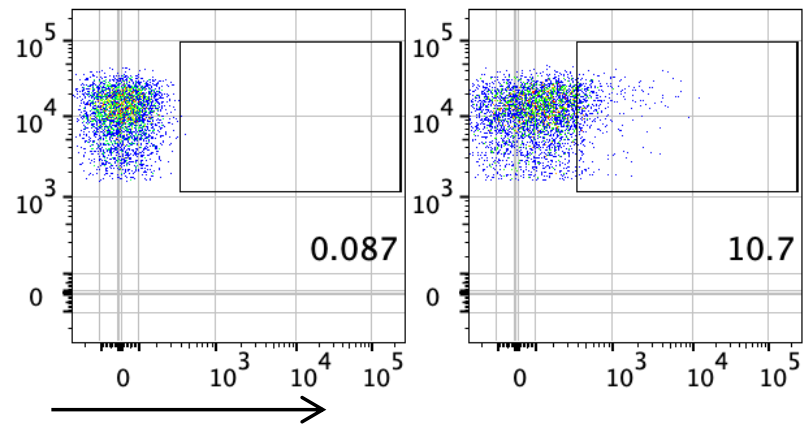

C

\%CD8 on CD45

4-1BB-PE
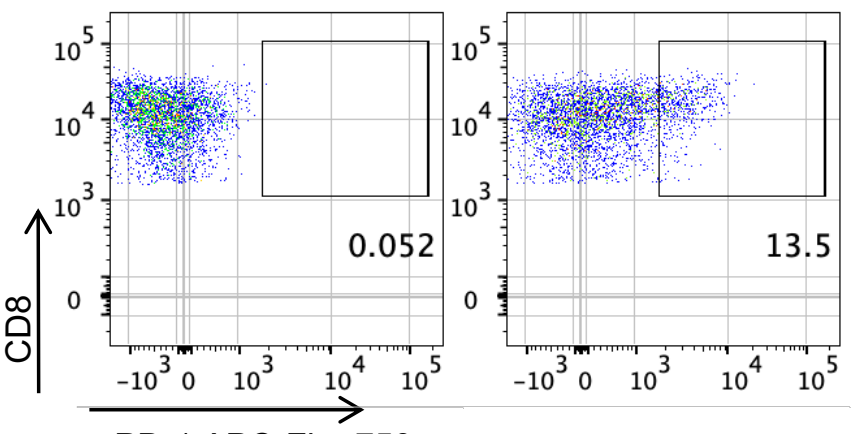

PD-1 APC-Fire 750

Figure 2. CD39 expression among CD8 TILs varies with lung cancer subtype. A) Representative flow cytometry staining of CD39, Tim-3, 4-1BB, and PD-1 on DAPI ${ }^{-} \mathrm{CD} 45^{+} \mathrm{CD}^{+} \mathrm{CD} 8^{+} \mathrm{T}$ cells from MSK 1105b. Left plot represents fluorescence minus one (FMO) and right plot represents the CD8 T cells stained with the indicated antibody. B-C) Violin plots of \%CD39+ (among CD8 ${ }^{+} \mathrm{T}$ cells) and \%CD8 (among CD45 ${ }^{+}$) for various histological subtypes/driver mutation categories among the 440-sample cohort. Dashed line indicates 23.3\% mean CD39 level for entire 440-sample cohort. Red bars indicate histological subtypes/driver mutations with above average \%CD39 values. 

(which was not certified by peer review) is the author/funder. All rights reserved. No reuse allowed without permission.

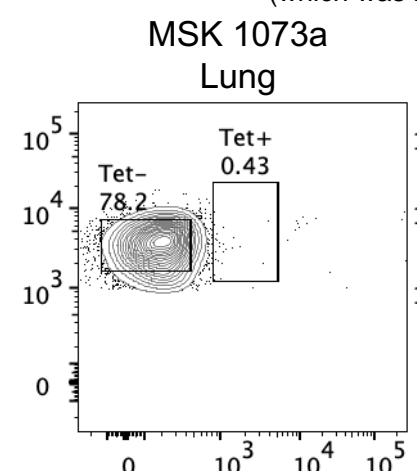

MSK 1329a

MSK 1089a

MSK 1393a

MSK 1430a

Pleural Effusion
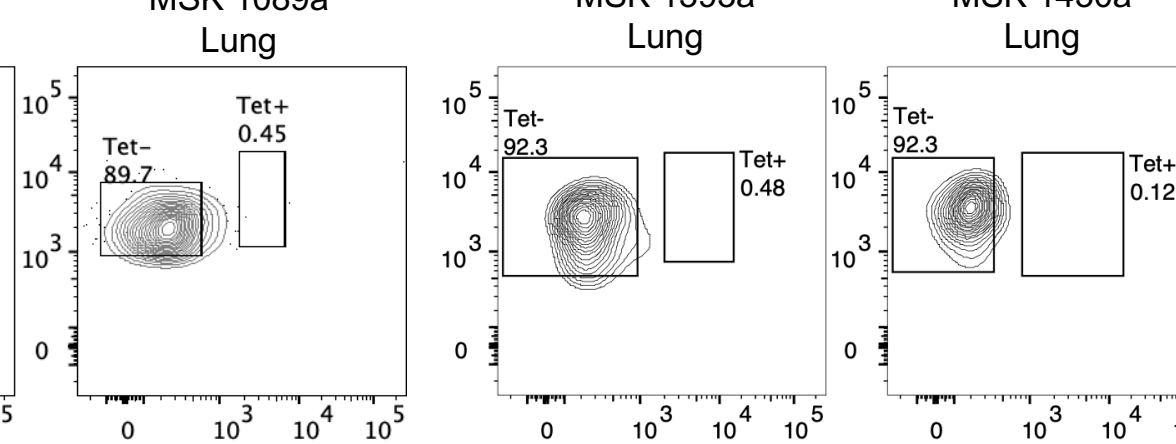

$0 \stackrel{1}{1}$
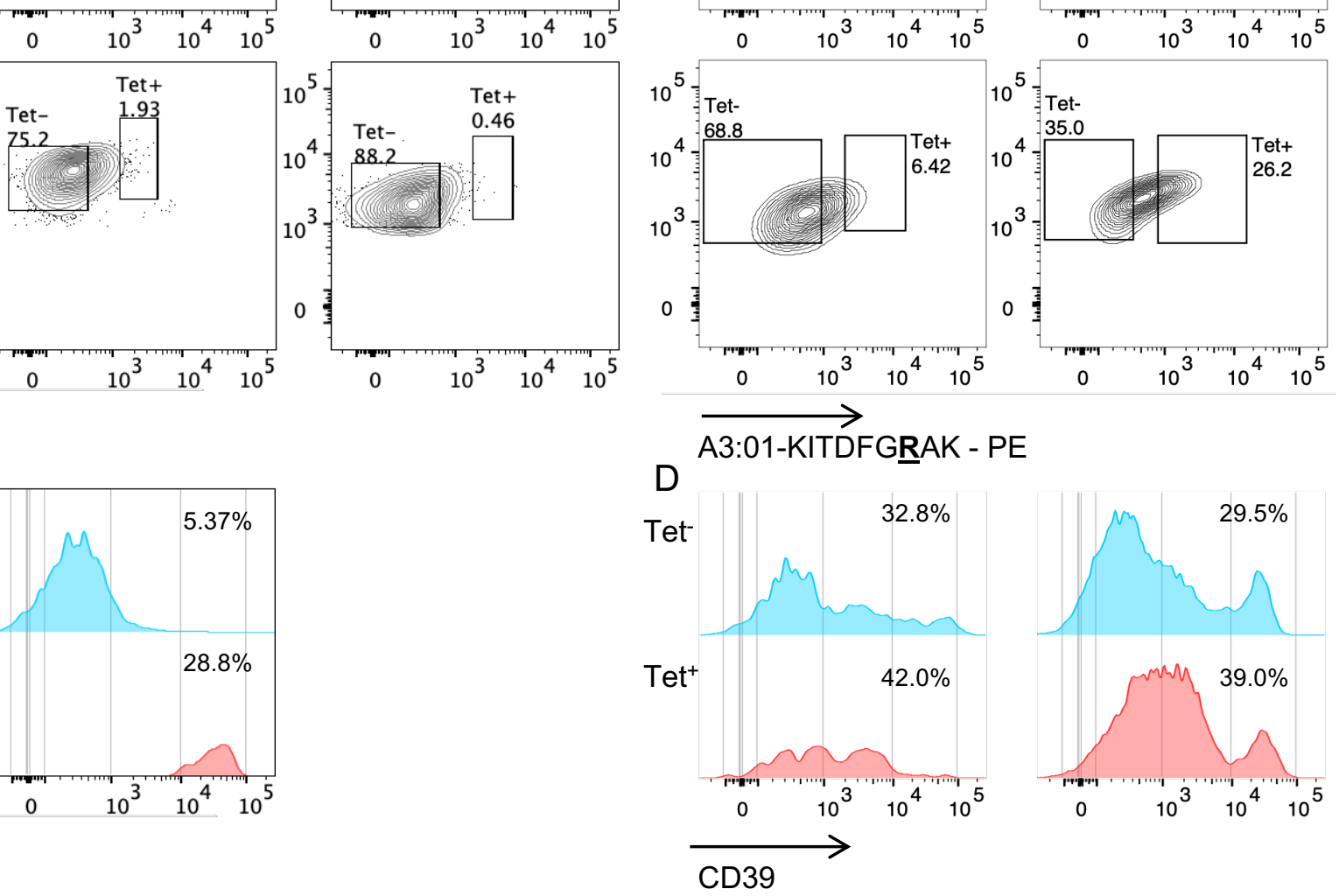

B

A3:01-AIKTSPKANK - PE
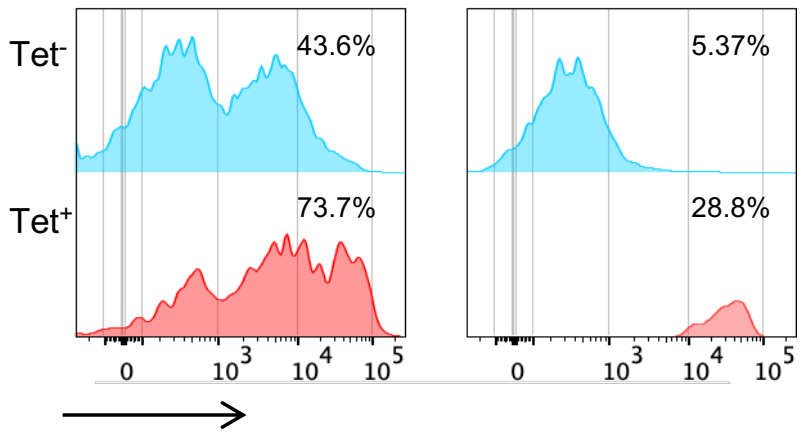

CD39
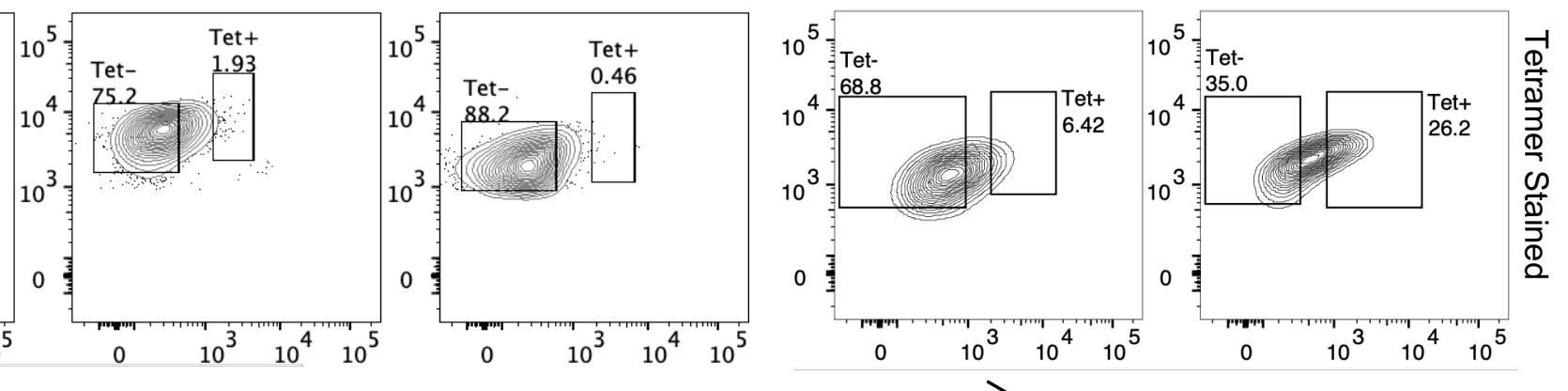

Figure 3. Tumor neoantigen-specific CD8 T cells express CD39. A) Flow cytometry plots from three patients with EGFR ex19 p.E746_A750del mutant lung cancer showing staining for A3:01: AIKTSPKANK tetramer. Top row depicts staining of A3:01: AIKTSPKANK tetramer negative control. Bottom row depicts staining of A3:01: AIKTSPKANK tetramer. B) Flow cytometry histogram showing \%CD39+ among tetramer ${ }^{-}$(et $^{-}$) and tetramer ${ }^{+}$ $\left(\mathrm{Tet}^{+}\right)$CD8 T cells. C) Flow cytometry plots from two patients with EGFR exon 21 L858R mutant lung cancer staining for A3:01: KITDFGRAK tetramer. Top row depicts staining of A3:01: KITDFGRAK tetramer negative control. Bottom row depicts staining of A3:01: KITDFGRAK tetramer. D) Flow cytometry histogram showing $\% \mathrm{CD} 9^{+}$among tetramer (Tet) and tetramer ${ }^{+}\left(\mathrm{Tet}^{+}\right)$CD8 T cells. 


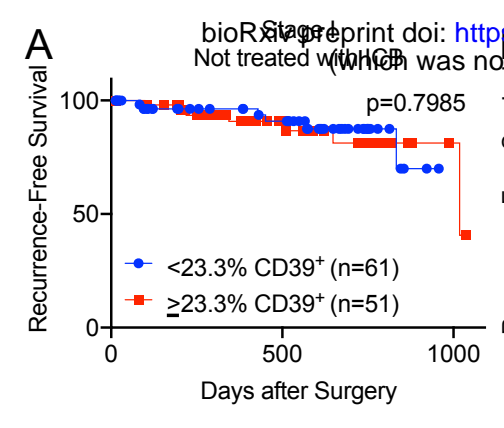

\begin{tabular}{|c|c|}
\hline Total & 22 \\
\hline \multicolumn{2}{|l|}{ Treatment } \\
\hline - Pembrolizumab & 11 \\
\hline - Nivolumab & 4 \\
\hline - Atezolizumab & 4 \\
\hline - Ipilimumab/ Nivolumab & 3 \\
\hline \multicolumn{2}{|l|}{ Histology/Driver Mutation } \\
\hline - KRAS Adenocarcinoma & 9 \\
\hline - EGFR Adenocarcinoma & 3 \\
\hline - Unknown Driver AdenoCa & 4 \\
\hline - Squamous & 3 \\
\hline - SCLC/LCLC & 3 \\
\hline
\end{tabular}
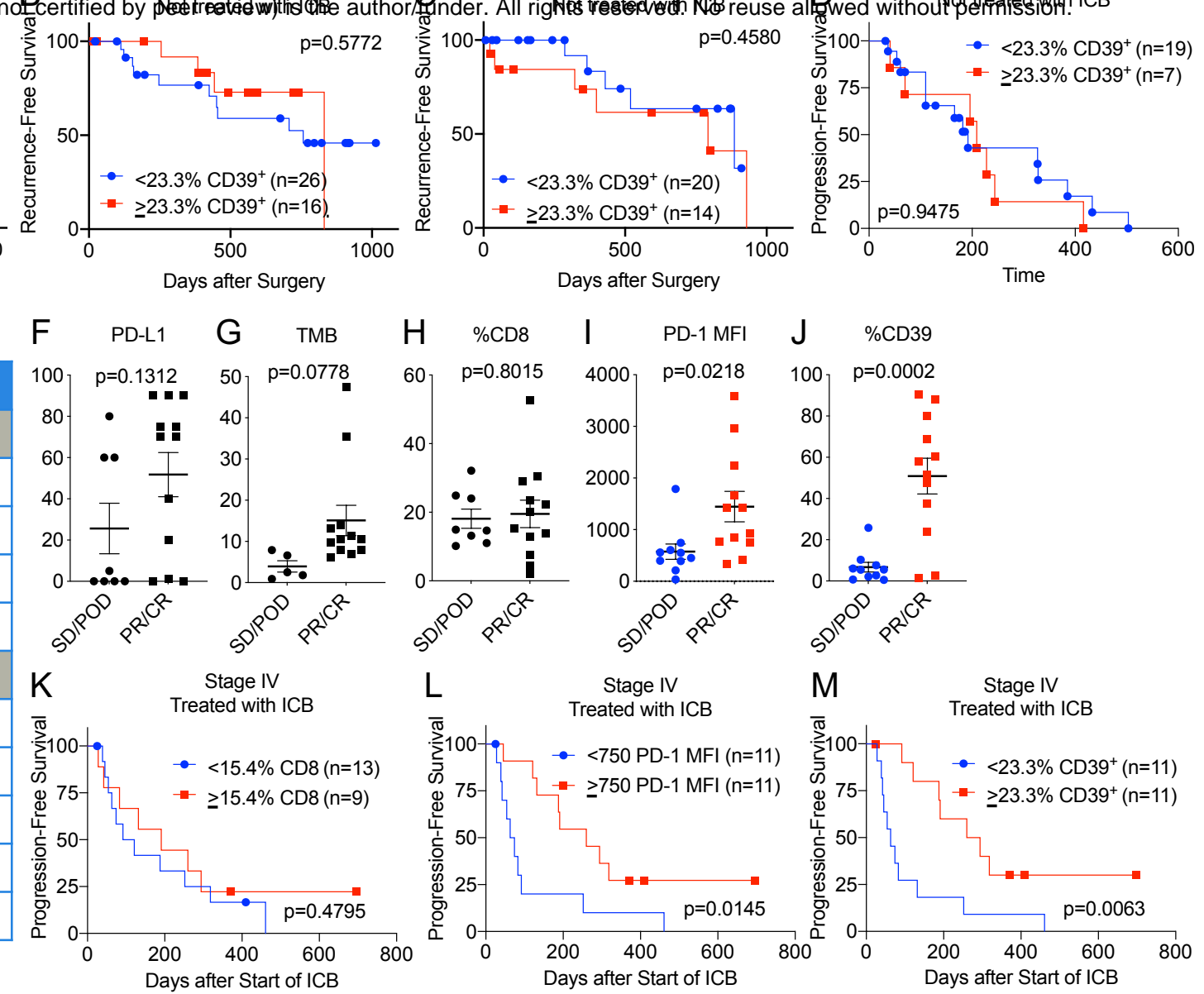

Figure 5. CD39 expression among CD8 tumor-infiltrating lymphocytes is not prognostic, but portends a beneficial outcome to immune checkpoint blockade in lung cancer. $\mathrm{A}-\mathrm{C}$ ) Kaplan-Meier curve of recurrencefree survival after resection with above or below mean \%CD39 (among CD8 T cells) for stage I, stage II, and stage IIIA lung cancer patients who did not receive ICB. Statistical significance determined by Mantel-Cox test. D) Kaplan-Meier curve of progression-free survival after resection with above or below mean \%CD39 (among CD8 T cells) for stage IV lung cancer patients who did not receive ICB. Statistical significance determined by Mantel-Cox test. E) Cohort of stage IV lung cancer patients who received ICB monotherapy. F-J) Tumor proportion score for PD-L1, tumor mutation burden, \%CD8 (among CD45 ), PD-1 mean fluorescence intensity (MFI) and \% CD39 (among CD8 T cells) among non-responders (stable disease/progression of disease, $\mathrm{SD} / \mathrm{POD}$ ) or responders (partial response/complete response, $\mathrm{PR} / \mathrm{CR}$ ) to ICB in the cohort described in E). K-M) Kaplan-Meier survival curve of progression-free survival patients described in cohort $E$ based on stratification for CD8, PD-1, and CD39. Statistical significance was determined by Mantel-Cox test. 
A bGBR
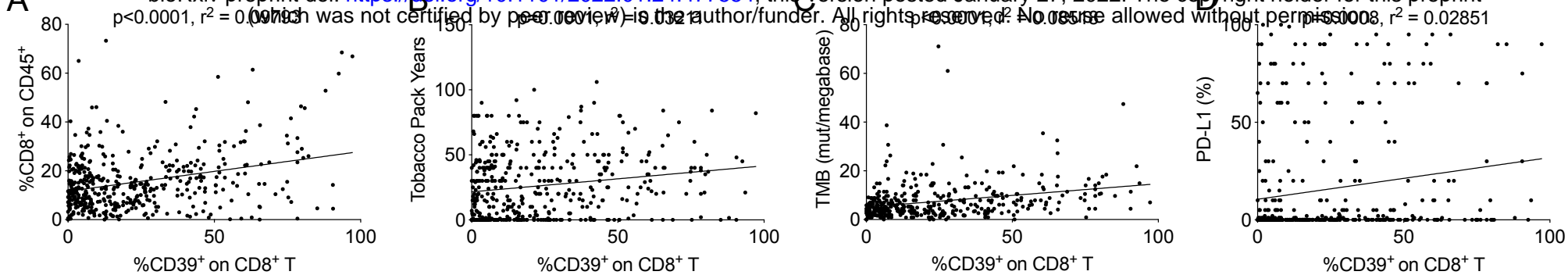

E CD39 Correlation with TMB and PD-L1
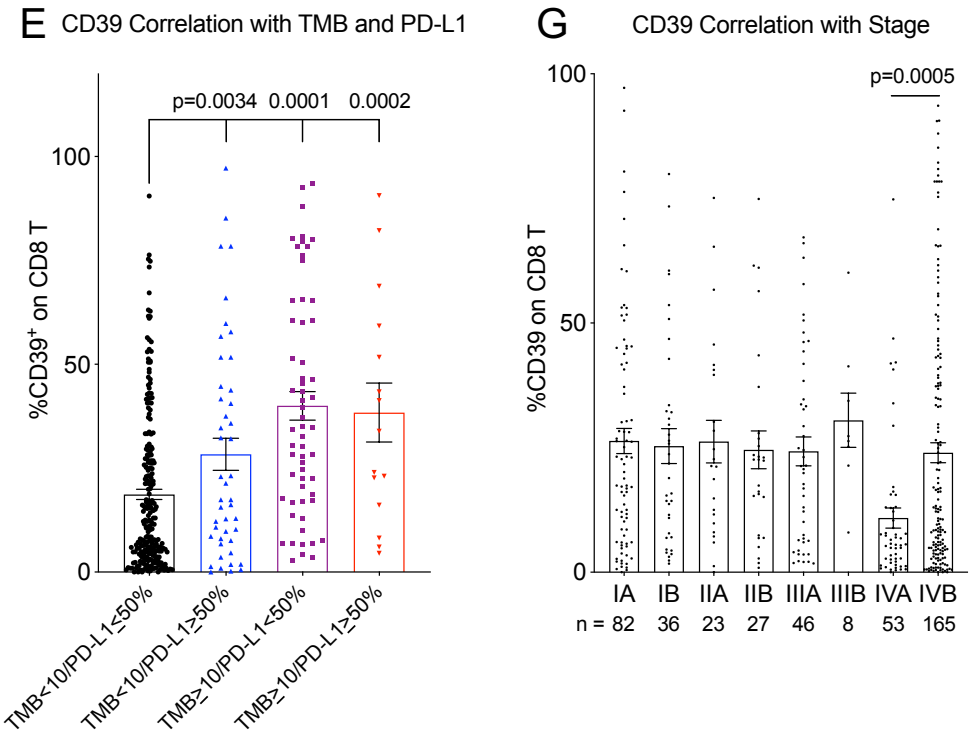

F CD8 Correlation with TMB and PD-L1

H CD39 Correlation with Anatomic Site
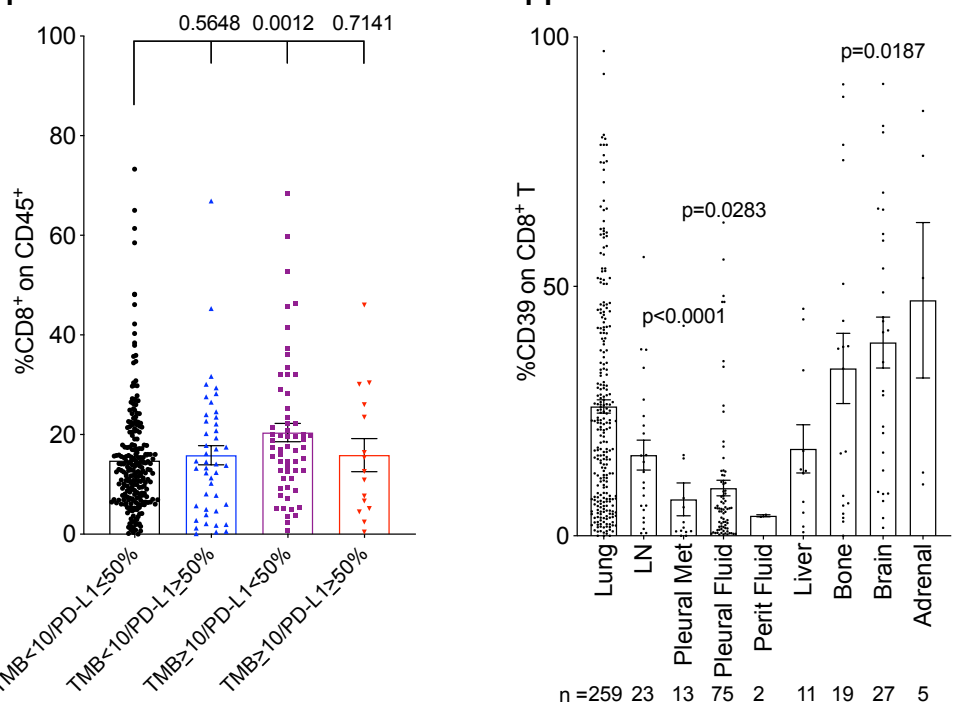

I

TMB (mutations/megabase)
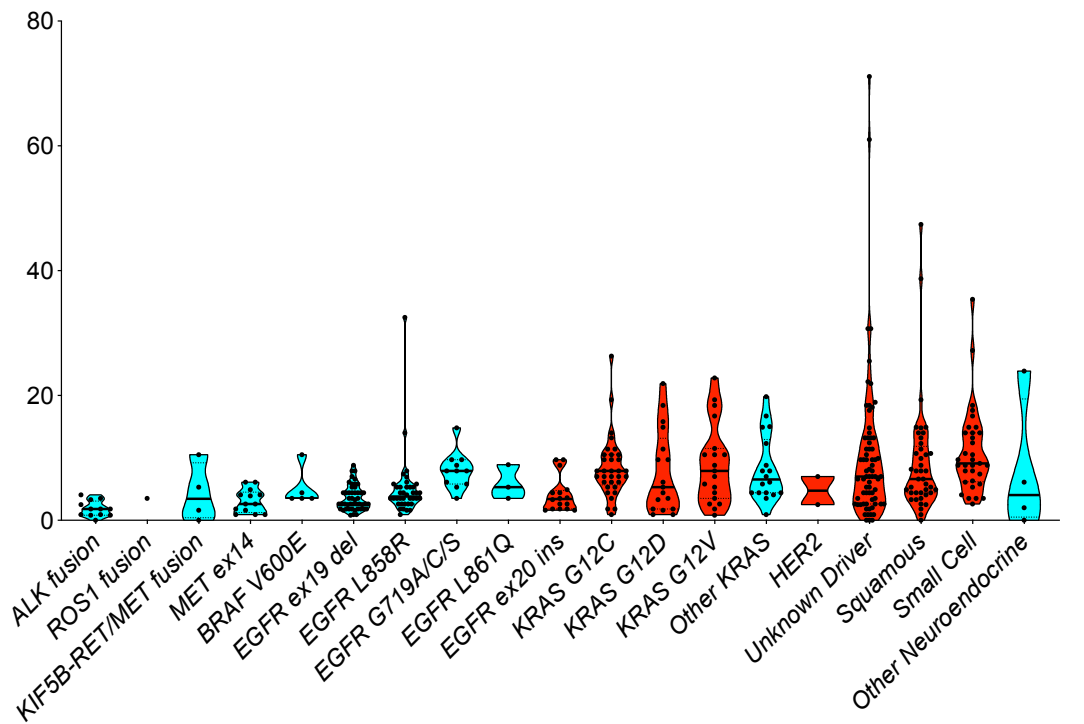

J Tumor Proportion PD-L1 (\%)

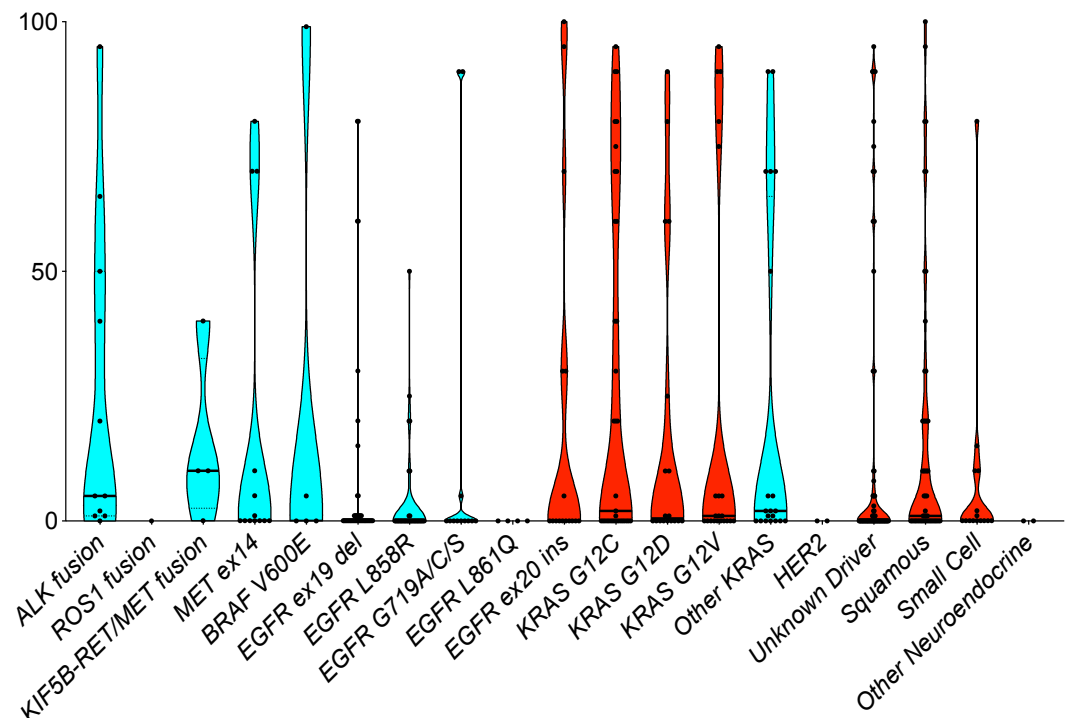

Figure S2. Clinical and molecular correlates of CD39 expression on CD8 tumor-infiltrating lymphocytes. A-D) Correlation of \%CD39 on CD8 T cells with \%CD8 (among CD45 ), tumor mutation burden, PD-L1 tumor proportion score, and smoking history. E-F) \%CD39 (among CD8 T cells) and \%CD8 (among CD45 ${ }^{+}$) on four subgroups based on cutoffs for TMB and PDL-1 in the 440-sample cohort. Statistical significance was assessed by two-tailed student's t-test. G) Correlation of \%CD39 on CD8 T by lung cancer stage. H) Correlation of \%CD39 on CD8 T by anatomic site of biospecimen. I-J) Correlation of tumor mutation burden and PD-L1 tumor proportion score with various histological subtypes/driver mutation categories among 440-sample cohort. Red bars indicate histological subtypes/driver mutations with above average \%CD39 values. 
